\title{
THE COSMIC EVOLUTION OF FERMI BL LACERTAE OBJECTS
}

\author{
M. Ajello ${ }^{1}$, R. W. Romani ${ }^{2}$, D. Gasparrini ${ }^{3,4}$, M. S. Shaw ${ }^{2}$, J. Bolmer ${ }^{5}$, G. Cotter ${ }^{6}$, J. Finke ${ }^{7}$, \\ J. Greiner ${ }^{8}$, S. E. Healey ${ }^{2}$, O. King ${ }^{9}$, W. MaX-Moerbeck ${ }^{9}$, P. F. Michelson ${ }^{2}$, W. J. Potter ${ }^{6}$, \\ A. RaU ${ }^{8}$, A. C. S. Readhead ${ }^{9}$, J. L. Richards ${ }^{9}$, AND P. SCHady ${ }^{8}$ \\ ${ }^{1}$ Space Sciences Laboratory, 7 Gauss Way, University of California, Berkeley, CA 94720-7450, USA; majello@ ssl.berkeley.edu \\ ${ }^{2}$ W. W. Hansen Experimental Physics Laboratory, Kavli Institute for Particle Astrophysics and Cosmology, Department of Physics \\ and SLAC National Accelerator Laboratory, Stanford University, Stanford, CA 94305, USA; rwr@ astro.stanford.edu, msshaw@ stanford.edu \\ ${ }^{3}$ Agenzia Spaziale Italiana (ASI) Science Data Center, I-00044 Frascati, Roma, Italy; gasparrini@ asdc.asi.it \\ ${ }^{4}$ Istituto Nazionale di Astrofisica-Osservatorio Astronomico di Roma, I-00040 Monte Porzio Catone, Roma, Italy \\ ${ }^{5}$ Physik Dept., Technische Universität München, James-Franck-Str., 85748 Garching, Germany \\ ${ }^{6}$ Department of Astrophysics, University of Oxford, Oxford OX1 3RH, UK \\ ${ }^{7}$ Space Science Division, Naval Research Laboratory, Washington, DC 20375-5352, USA \\ ${ }^{8}$ Max-Planck Institut für extraterrestrische Physik, D-85748 Garching, Germany \\ ${ }^{9}$ Department of Astronomy, California Institute of Technology, Pasadena, CA 91125, USA \\ Received 2013 June 3; accepted 2013 September 26; published 2013 December 13
}

\begin{abstract}
Fermi has provided the largest sample of $\gamma$-ray-selected blazars to date. In this work we use a uniformly selected set of 211 BL Lacertae (BL Lac) objects detected by Fermi during its first year of operation. We obtained redshift constraints for 206 out of the 211 BL Lac objects in our sample, making it the largest and most complete sample of BL Lac objects available in the literature. We use this sample to determine the luminosity function of BL Lac objects and its evolution with cosmic time. We find that for most BL Lac classes the evolution is positive, with a space density peaking at modest redshift $(z \approx 1.2)$. Low-luminosity, high-synchrotron-peaked (HSP) BL Lac objects are an exception, showing strong negative evolution, with number density increasing for $z \lesssim 0.5$. Since this rise corresponds to a drop-off in the density of flat-spectrum radio quasars (FSRQs), a possible interpretation is that these HSPs represent an accretion-starved end state of an earlier merger-driven gas-rich phase. We additionally find that the known BL Lac correlation between luminosity and photon spectral index persists after correction for the substantial observational selection effects with implications for the so-called "blazar sequence." Finally, by estimating the beaming corrections to the luminosity function, we find that BL Lac objects have an average Lorentz factor of $\gamma=6.1_{-0.8}^{+1.1}$, and that most are seen within $10^{\circ}$ of the jet axis.
\end{abstract}

Key words: cosmology: observations - diffuse radiation - galaxies: active - galaxies: jets - gamma rays: diffuse background - surveys

Online-only material: color figures

\section{INTRODUCTION}

BL Lacertae (BL Lac) objects are a subpopulation of blazars, an extreme class of active galactic nuclei (AGNs), displaying highly variable emission likely due to a relativistic jet pointing close to our line of sight (e.g., Blandford \& Rees 1978). They are distinguished from their siblings, the flat-spectrum radio quasars (FSRQs) by an optical spectrum lacking any emission lines with an equivalent width $>5 \AA$ (e.g., Urry \& Padovani 1995; Marcha et al. 1996). The optical spectra of BL Lac objects are power-law-dominated, indicating either especially strong nonthermal continuum (jet aligned very close to our line of sight) or unusually weak thermal disk/broadline emission (plausibly attributed to low accretion activity; Giommi et al. 2012).

The synchrotron component ${ }^{10}$ of BL Lac objects shows a range of peak frequencies from $v \approx 10^{13} \mathrm{~Hz}$ up to $v \approx 10^{17} \mathrm{~Hz}$ (e.g., Ackermann et al. 2011). At the high end, these synchrotron peaks imply that BL Lac objects are able to accelerate electrons beyond $100 \mathrm{TeV}$ (e.g., Costamante et al. 2001; Tavecchio et al.

\footnotetext{
${ }^{10}$ BL Lac objects and blazars in general can be classified according to the frequency, in the rest frame, of the peak of the synchrotron component as low-synchrotron-peaked (LSP, $v_{\text {peak }}<10^{14} \mathrm{~Hz}$ ), intermediate-synchrotronpeaked (ISP, $10^{14}<v_{\text {peak }}<10^{15} \mathrm{~Hz}$ ), and high-synchrotron-peaked (HSP, $\left.v_{\text {peak }}>10^{15} \mathrm{~Hz}\right)$.
}

2011), making BL Lac objects among the most powerful accelerators in the universe.

The lack of strong emission lines hampers traditional optical spectroscopic measurements of the redshifts of most BL Lac objects. Indeed, roughly 55\% of the 395 BL Lac objects detected in the second Fermi AGN catalog (2LAC; Ackermann et al. 2011) lacked a spectroscopic redshift. This limitation is also serious at lower frequencies (Padovani et al. 2007), and the large redshift incompleteness of most BL Lac samples has so far hampered the determination of a reliable luminosity function (LF). In turn, this handicaps studies of the growth and evolution of BL Lac objects in the universe and the relationship between BL Lac objects and the FSRQ class.

While it is clear that FSRQs evolve positively at all frequencies (i.e., there were more blazars in the past; Dunlop \& Peacock 1990) up to a redshift cutoff that depends on luminosity (e.g., Padovani et al. 2007; Wall 2008; Ajello et al. 2009; Ajello et al. 2012), the evolution of BL Lac objects remains a matter of debate. Indeed, various studies have found that BL Lac objects evolved negatively (e.g., Rector et al. 2000; Beckmann et al. 2003), positively (e.g., Marcha \& Caccianiga 2013), or not at all (Caccianiga et al. 2002; Padovani et al. 2007). These discrepancies might be due to small samples, biases in the set of BL Lac objects, and substantial redshift incompleteness in these works.

At gamma-ray energies the need for a reliable LF is particularly acute. Indeed, the present lack of a secure LF makes 
it impossible to estimate the contribution of faint (below detection threshold) BL Lac objects to the isotropic gamma-ray background (IGRB; Abdo et al. 2010b). At GeV-TeV energies BL Lac objects are characterized by a harder spectrum than FSRQs and are found to outnumber (by a factor $>3$ ) the latter particularly above $10 \mathrm{GeV}$ (Abdo et al. 2010c). Thus at high energies these sources may well dominate the cosmic gamma-ray background.

Thanks to its excellent sensitivity, the Large Area Telescope (LAT) on board Fermi has detected $\sim 395$ BL Lac objects in the first two years of operations (Ackermann et al. 2011). To study this sample many different techniques have been employed to obtain redshift estimates or constraints for these blazars (see Rau et al. 2012; Shaw et al. 2013a, 2013b), yielding the rather surprising detection of several BL Lac objects up to redshift $z \approx 2$. These high $-z$ objects often show a very hard (photon index of 2) GeV spectrum, making them the most luminous BL Lac objects of the HSP kind ever detected. How these objects fit within the scheme of the blazar population and blazar sequence is still highly debated (Padovani et al. 2012; Ghisellini et al. 2012).

In this work we study the cosmological properties of BL Lac objects, focusing on a complete set of 211 BL Lac objects detected by Fermi-LAT during the first year of operation (Abdo et al. 2010d). Using the full range of techniques (see Rau et al. 2012; Shaw et al. 2013b), we have obtained spectroscopic redshifts or limits for the great majority $(\sim 98 \%)$ of the sources. This has let us derive the first detailed models for the LF and evolution of BL Lac objects at $\mathrm{GeV}$ energies. The large sample size and unusually high redshift completeness allow new inferences about the nature of the BL Lac population as a whole. This article is organized as follows: Sections 2 and 3 present the properties of the sample, discuss the available redshift constraints and describe the method used to derive the LF. The results are presented and discussed in Sections 4-6. Throughout this article, a standard concordance cosmology was assumed $\left(\mathrm{H}_{0}=71 \mathrm{~km} \mathrm{~s}^{-1} \mathrm{Mpc}^{-1}, \Omega_{\mathrm{M}}=1-\Omega_{\Lambda}=0.27\right)$.

\section{THE SAMPLE}

The first Fermi-LAT catalog (1FGL; Abdo et al. 2010a) presented more than 1400 sources detected by Fermi-LAT during its first year of operation. The first LAT AGN catalog (1LAC; Abdo et al. 2010d) associates 700 of the high-latitude 1FGL sources $\left(|b| \geqslant 10^{\circ}\right)$ with AGNs of various types, most of which are blazars. The sample used for this analysis consists of sources detected by the pipeline developed by Abdo et al. (2010c) with a test statistic ${ }^{11}$ (TS) greater (or equal) than 50 and with $|b| \geqslant 15^{\circ}$. For these sample cuts we have produced a set of Monte Carlo simulations that can be used to determine and correct for the selection effects. This sample contains 486 objects, 211 of which are classified as BL Lac objects in 1LAC. The composition of this sample is reported in Table 1. The source classifications reported in Table 1 are originally drawn from the 1LAC and 2LAC catalogs (Abdo et al. 2010d),

\footnotetext{
11 The test statistic (or TS) is defined as TS $=-2\left(\ln L_{0}-\ln L_{1}\right)$, where $L_{0}$ and $L_{1}$ are the likelihoods of the background (null hypothesis) and the hypothesis being tested (e.g., source plus background). According to Wilks (1938), the TS is expected to be asymptotically distributed as $\chi_{n}^{2}$ in the null hypothesis, where $n$ is the additional number of free parameters that are optimized for the alternative hypothesis. Given the 4 degrees of freedom required for source detection (position and spectral parameters), a TS of 50 corresponds to $\sim 6.3 \sigma$ of a Gaussian distribution.
}

Table 1

Composition of the $|b| \geqslant 15^{\circ}$, TS $\geqslant 50$ Sample Used in This Analysis

\begin{tabular}{lc}
\hline \hline Class & No. of Objects \\
\hline Total & 486 \\
BL Lac objects & 211 \\
FSRQs & 186 \\
Pulsars & 31 \\
Dropped by 2FGL & 2 \\
Other $^{\mathrm{a}}$ & 33 \\
Unassociated sources & 23 \\
\hline
\end{tabular}

Notes. ${ }^{\text {a }}$ Includes starburst galaxies, LINERS, narrow-line Seyfert 1 objects, Seyfert galaxy candidates, and Fermi sources with a radio counterpart, but no optical type or redshift measurement.

and they have been complemented with newer observations reported in Shaw et al. (2012) and Shaw et al. (2013b).

The 211 BL Lac objects detected by Fermi with TS $\geqslant 50$, $|b| \geqslant 15^{\circ}$ constitute the sample that will be used in this analysis. All these objects are reported together with their properties in Table 5 in Appendix A.1. We note that fluxes and photon indices reported there are those measured with the pipeline developed by Abdo et al. (2010c) and thus, while compatible with the values reported in the 1FGL catalog (Abdo et al. 2010a), they are not exactly the same. These values are meant to be used with the results of the Monte Carlo simulations to correctly account for selection effects (see Sections 4 and 5 in Abdo et al. 2010c).

Of the 38 sources remaining unclassified in 1FGL, 3 objects now have pulsar identifications, 2 sources have been dropped as spurious composites, and 10 are flagged as pulsar candidates based on their variability and spectral properties (Ackermann et al. 2012a). This leaves 23 objects that might be blazars yet to be identified. Recent radio observations (Petrov et al. 2013) find compact source counterparts for 11 of these, so it is likely that these 11 represent missing BL Lac objects. Moreover, when cross-correlating the list of 23 objects with the WISE sources whose colors are typical of blazars (Massaro et al. 2012; D'Abrusco et al. 2012), we found an additional 8 blazar candidates. Thus a total of 19 sources display properties of blazars on the basis of their IR colors or radio properties. Conservatively we assume that all these sources might be BL Lac objects, and that the incompleteness (due to missing identification) in our BL Lac sample is $19 / 211=9 \%$. The total incompleteness (due to missing redshifts and identifications) is thus $\sim 11 \%$. As it will be shown later this incompleteness does not constitute a problem for the analysis.

\section{ANALYSIS}

\subsection{Method}

In order to derive the LF of BL Lac objects, we rely on the maximum likelihood (ML) method first introduced by Marshall et al. (1983) and used recently for the study of blazars detected by Swift (Ajello et al. 2009) and FSRQs detected by Fermi (Ajello et al. 2012). The aim of this analysis is to determine the space density of BL Lac objects as a function of rest-frame $0.1-100 \mathrm{GeV}$ luminosity $\left(L_{\gamma}\right)$, redshift $(z)$ and photon index $(\Gamma)$ by fitting to the functional form:

$$
\frac{\partial^{3} N}{\partial L_{\gamma} \partial z \partial \Gamma}=\frac{\partial^{3} N}{\partial L_{\gamma} \partial V \partial \Gamma} \times \frac{d V}{d z}=\Phi\left(L_{\gamma}, V(z), \Gamma\right) \times \frac{d V}{d z},
$$


where $\Phi\left(L_{\gamma}, V(z), \Gamma\right)$ is the LF, and $d V / d z$ is the comoving volume element per unit redshift and unit solid angle (see, e.g., Hogg 1999).

The best-fit LF is found by comparing, through an ML estimator, the number of expected objects (for a given model LF) to the observed number while accounting for selection effects in the detection of gamma-ray sources. In this method, the space of luminosity, redshift, and photon index are divided into small intervals of size $d L_{\gamma} d z d \Gamma$. In each element, the expected number of blazars with luminosity $L_{\gamma}$, redshift $z$, and photon index $\Gamma$ is

$$
\begin{aligned}
\lambda\left(L_{\gamma}, z, \Gamma\right) d L_{\gamma} d z d \Gamma=\Phi & \left(L_{\gamma}, V(z), \Gamma\right) \cdot \Omega\left(L_{\gamma}, z, \Gamma\right) \\
& \times \frac{d V}{d z} d L_{\gamma} d z d \Gamma,
\end{aligned}
$$

where $\Omega\left(L_{\gamma}, z, \Gamma\right)$ is the sky coverage and represents the probability of detecting a blazar with luminosity $L_{\gamma}$, redshift $z$, and photon index $\Gamma$ in this survey. This probability was derived for the sample used here by Abdo et al. (2010c), and the reader is referred to that article for more details. With sufficiently fine sampling of the $L_{\gamma}-z-\Gamma$ space, the infinitesimal element will either contain 0 or $1 \mathrm{BL}$ Lac. In this regime one has a likelihood function based on joint Poisson probabilities:

$$
\begin{aligned}
L= & \prod_{i} \lambda\left(L_{\gamma, i}, z_{i}, \Gamma_{i}\right) d L_{\gamma} d z d \Gamma e^{-\lambda\left(L_{\gamma, i}, z_{i}, \Gamma_{i}\right) d L_{\gamma} d z d \Gamma} \\
& \times \prod_{j} e^{-\lambda\left(L_{\gamma, j}, z_{j}, \Gamma_{j}\right) d L_{\Gamma} d z d \Gamma} .
\end{aligned}
$$

This is the combined probability of detecting one blazar in each bin of $\left(L_{\gamma, i}, z_{i}, \Gamma_{i}\right)$ populated by one observed Fermi BL Lac and zero BL Lac objects for all other $\left(L_{\gamma, j}, z_{j}, \Gamma_{j}\right)$. Transforming to the standard expression $S=-2 \ln L$ and dropping terms which are not model dependent, we obtain:

$$
\begin{aligned}
S= & -2 \sum_{i} \ln \frac{\partial^{3} N}{\partial L_{\gamma} \partial z \partial \Gamma}+2 \int_{\Gamma_{\min }}^{\Gamma_{\max }} \int_{L_{\gamma, \min }}^{L_{\gamma, \max }} \\
& \times \int_{z_{\min }}^{z_{\max }} \lambda\left(L_{\gamma}, \Gamma, z\right) d L_{\gamma} d z d \Gamma .
\end{aligned}
$$

The limits of integration of Equation (4) and subsequent equations, unless otherwise stated, are $L_{\gamma, \min }=7 \times 10^{43} \mathrm{erg} \mathrm{s}^{-1}$, $L_{\gamma, \max }=10^{52} \mathrm{erg} \mathrm{s}^{-1}, z_{\min }=0.03, z_{\max }=6, \Gamma_{\min }=1.45$, and $\Gamma_{\max }=2.80$. The results of this analysis are independent of the choice of the maximum redshift and luminosity. All other limits correspond to those spanned by the set of sources analyzed here. The best-fit parameters are determined by minimizing, ${ }^{12} S$ and the associated $1 \sigma$ errors are computed via bootstrap analysis (see below). While computationally intensive, Equation (4) has the advantage that each source has its appropriate individual detection efficiency and $k$ correction ${ }^{13}$ treated independently.

To test whether the best-fit LF provides a good description of the data, we compare the observed redshift, luminosity, index, and source count distributions against the prediction of the LF. The first three distributions can be obtained from the LF as

$$
\frac{d N}{d z}=\int_{\Gamma_{\min }}^{\Gamma_{\max }} \int_{L_{\gamma, \min }}^{L_{\gamma, \max }} \lambda\left(L_{\gamma}, \Gamma, z\right) d L_{\gamma} d \Gamma
$$

\footnotetext{
12 The MINUIT minimization package, embedded in ROOT (root.cern.ch), has been used for this purpose.

13 The $k$ correction is the ratio of source rest-frame luminosity to observed luminosity and allows the transformation of an observed luminosity into a rest-frame one.
}

$$
\begin{gathered}
\frac{d N}{d L_{\gamma}}=\int_{\Gamma_{\min }}^{\Gamma_{\max }} \int_{z_{\min }}^{z_{\max }} \lambda\left(L_{\gamma}, \Gamma, z\right) d z d \Gamma, \\
\frac{d N}{d \Gamma}=\int_{L_{\gamma, \min }}^{L_{\gamma, \max }} \int_{z_{\min }}^{z_{\max }} \lambda\left(L_{\gamma}, \Gamma, z\right) d L_{\gamma} d z,
\end{gathered}
$$

where the limits of integration are the same as in Equation (4). The source count distribution can be derived as

$$
N(>F)=\int_{\Gamma_{\min }}^{\Gamma_{\max }} \int_{z_{\min }}^{z_{\max }} \int_{L_{\gamma}(z, F)}^{L_{\gamma, \max }} \Phi\left(L_{\gamma}, V(z), \Gamma\right) \frac{d V}{d z} d \Gamma d z d L_{\gamma}
$$

where $L_{\gamma}(z, F)$ is the luminosity of a source at redshift $z$ having a flux of $F$.

To display the LF we rely on the " $N^{\text {obs }} / N^{\text {mdl " }}$ method devised by La Franca \& Cristiani (1997) and Miyaji et al. (2001) and employed in several recent works (e.g., La Franca et al. 2005; Hasinger et al. 2005). Once a best-fit function for the LF has been found, it is possible to determine the value of the observed LF in a given bin of luminosity and redshift:

$$
\Phi\left(L_{\gamma, i}, V\left(z_{i}\right), \Gamma_{i}\right)=\Phi^{\mathrm{mdl}}\left(L_{\gamma, i}, V\left(z_{i}\right), \Gamma_{i}\right) \frac{N_{i}^{\mathrm{obs}}}{N_{i}^{\mathrm{mdl}}},
$$

where $L_{\gamma, i}, z_{i}$, and $\Gamma_{i}$ are the luminosity, redshift, and photon index of the $i^{\text {th }}$ bin; $\Phi^{\mathrm{mdl}}\left(L_{\gamma, i}, V\left(z_{i}\right), \Gamma_{i}\right)$ is the best-fit LF model; and $N_{i}^{\text {obs }}$ and $N_{i}^{\mathrm{mdl}}$ are the observed and the predicted numbers of BL Lac objects in that bin. These two techniques (the

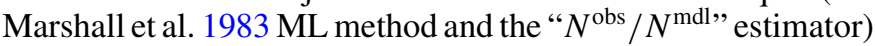
provide a minimally biased estimate of the LF (cf. Miyaji et al. 2001).

\subsection{Parameterization of the Luminosity Function}

We model the intrinsic distribution of photon indices with a Gaussian, which implies that for a given redshift $z$ and luminosity $L_{\gamma}$ the LF is

$$
\Phi\left(L_{\gamma}, z, \Gamma\right) \propto e^{-\frac{\left(\Gamma-\mu\left(L_{\gamma}\right)\right)^{2}}{2 \sigma^{2}}}
$$

where $\mu$ and $\sigma$ are, respectively, the Gaussian mean and dispersion. To test a possible correlation of the photon index with luminosity, as previously noted in the literature (see, e.g., Ghisellini et al. 2009; Meyer et al. 2012), we allow the mean ${ }^{14}$ $\mu$ to be a function of the source luminosity:

$$
\mu\left(L_{\gamma}\right)=\mu^{*}+\beta \times\left(\log _{10}\left(L_{\gamma}\right)-46\right) .
$$

The LF at redshift $z=0$ is modeled as a smoothly joined double power law multiplied by the photon index distribution of Equation (10):

$$
\begin{aligned}
\Phi\left(L_{\gamma}, z=0, \Gamma\right)= & \frac{A}{\ln (10) L_{\gamma}}\left[\left(\frac{L_{\gamma}}{L_{*}}\right)^{\gamma_{1}}\right. \\
& \left.+\left(\frac{L_{\gamma}}{L_{*}}\right)^{\gamma 2}\right]^{-1} \cdot e^{-\frac{\left(\Gamma-\mu\left(L_{\gamma}\right)\right)^{2}}{2 \sigma^{2}}} .
\end{aligned}
$$

To parameterize the evolution of the LF we employ three commonly assumed evolutionary trends: a pure density evolution

\footnotetext{
14 We also tested a scenario for which $\sigma$ depends on the source luminosity or
} the redshift, but we did not find any evidence for such trends. 
(PDE), a pure luminosity evolution (PLE), and a luminositydependent density evolution (LDDE).

For both the PDE and PLE case we rely on an evolutionary factor defined as

$$
e(z)=(1+z)^{k_{d}} e^{z / \xi},
$$

where

$$
k_{d}=k^{*}+\tau \times\left(\log _{10}\left(L_{\gamma}\right)-46\right) .
$$

For the PDE the evolution is defined as

$$
\Phi\left(L_{\gamma}, z, \Gamma\right)=\Phi\left(L_{\gamma}, z=0, \Gamma\right) \times e(z),
$$

while for the PLE case it is

$$
\Phi\left(L_{\gamma}, z, \Gamma\right)=\Phi\left(L_{\gamma} / e(z), \Gamma\right)
$$

The PLE and PDE models have 10 free parameters $\left(A, \gamma_{1}\right.$, $L_{*}, \gamma_{2}, k^{*}, \tau, \xi, \mu^{*}, \beta$, and $\sigma$ ).

For the LDDE we adopt the same parameterization reported in Ajello et al. (2012):

$$
\Phi\left(L_{\gamma}, z, \Gamma\right)=\Phi\left(L_{\gamma}, z=0, \Gamma\right) \times e\left(z, L_{\gamma}\right)
$$

where

$$
\begin{gathered}
e\left(z, L_{\gamma}\right)=\left[\left(\frac{1+z}{1+z_{c}\left(L_{\gamma}\right)}\right)^{p 1\left(L_{\gamma}\right)}+\left(\frac{1+z}{1+z_{c}\left(L_{\gamma}\right)}\right)^{p 2}\right]^{-1} \\
z_{c}\left(L_{\gamma}\right)=z_{c}^{*} \cdot\left(L_{\gamma} / 10^{48}\right)^{\alpha} \\
p 1\left(L_{\gamma}\right)=p 1^{*}+\tau \times\left(\log _{10}\left(L_{\gamma}\right)-46\right)
\end{gathered}
$$

Here, $\Phi\left(L_{\gamma}, z=0, \Gamma\right)$ is the same double power law used in Equation (12), and $z_{c}\left(L_{\gamma}\right)$ corresponds to the (luminositydependent) redshift where the evolution changes sign (positive to negative), with $z_{c}^{*}$ being the redshift peak for a BL Lac with a luminosity of $10^{48} \mathrm{erg} \mathrm{s}^{-1}$. The LDDE model has a total of 12 free parameters $\left(A, \gamma_{1}, L_{*}, \gamma_{2}, z_{c}^{*}, p 1^{*}, \tau, p 2, \alpha, \mu^{*}, \beta\right.$, and $\left.\sigma\right)$. Note that the evolutionary term $e\left(z, L_{\gamma}\right)$ in Equation (18) is not equal to one at redshift zero (see also Section 4.2).

\subsection{Dealing with Redshift Constraints}

Only 103 of the 211 BL Lac objects in our sample have a spectroscopic redshift measurement (Ackermann et al. 2011). However, for another $104 \mathrm{BL}$ Lac objects we were able to provide quantitative constraints on the redshift. The constraints are as follows.

1. Photometric redshift estimates. The neutral hydrogen along the line of sight to the source efficiently absorbs photons with a rest-frame wavelength blue-wards of the Lyman limit. This results in a flux depression that can be used to estimate the absorber's redshift via spectral energy distribution (SED) template fitting. The absence of any drop-out provides an upper limit to the source redshift limited by the bluest available pass band (e.g., $z \leqslant 1.3$ based on Swift/UVOT in the study of Rau et al. 2012). In our sample, three sources have a photometric redshift estimate, while 34 have a photometric-redshift upper limit.
2. Redshift lower limits via intervening absorption systems. Metal line absorption systems (i.e., Mg II, Fe II, C IV, etc.) in the optical spectra caused by intervening systems provide a firm lower limit to the source redshift (Shaw et al. 2013b). In our sample, 39 sources have a spectroscopic redshift lower limit.

3. Spectroscopic redshift upper limits. Shaw et al. (2013b) used the absence of individual Lyman- $\alpha$ absorptions to provide statistically based upper limits for all the BL Lac objects without redshifts. As reported there, the exclusion $z_{\max }$ falls in the $1.65<z<3.0$ range. Although not as constraining as the UV-based SED bounds from Rau et al. (2012), we can extract these limits for all objects with spectra. All but five of our BL Lac objects were in the Shaw et al. (2013b) sample and thus have a $z_{m} a x$ estimate.

4. Host galaxy spectral fitting. According to, for example, Urry et al. (2000) and Sbarufatti et al. (2005), BL Lac objects are hosted by giant ellipticals with bright absolute magnitude of $M_{R}=-22.9 \pm 0.5$. If one assumes that these objects are standard candles, then the host non-detection places a lower limit on the source redshift. Shaw et al. (2013b) have improved this technique by fitting spectral templates of elliptical galaxies to their BL Lac optical spectra and recalibrating the host magnitudes against the spectroscopically measured set. For each trial redshift $z_{i}$ they are able to test the hypotheses of whether the optical spectrum is compatible (aside from the featureless BL Lac emission) with the red-shifted emission of the host galaxies. Thus, for every object they are able to provide exclusion probabilities for the source redshift as a function of redshift. Again, all but 5 of our BL Lac objects lacking spectroscopic redshifts have exclusion probabilities from Shaw et al. (2013b).

The five sources not included in Shaw et al. (2013b) and thus without redshift constraints are 1FGL J0006.9+4652, 1FGL J0322.1+2336, 1FGL J0354.6+8009, 1FGL J1838.6+4756, and 1FGL J2325.8-4043. All available constraints (with the exception of the exclusion functions) are listed in Table 5 in Appendix A.1. For each source, the available redshift constraints are combined. The most constraining cases are those where there is either a spectroscopic redshift lower limit (always coupled to a $z_{\max }$ limit) or a photometric upper limit (typically $z \lesssim 1.3$ ). Lower and upper limits on the redshift are treated as step functions, and we tested that the results reported in the next sections are robust against the exclusion of a fraction $(\sim 10 \%)$ of these limits.

For each object, we combine these constraints to produce the observationally allowed probability density function (PDF) for the source redshift. However, for the LF analysis we need the redshift PDF (subject to these observational constraints) for the source as a representative member of the Fermi-detected BL Lac objects. Accordingly, we assume a prior function that represents the $d N / d z$ distribution if one could measure the spectroscopic redshift for all the BL Lac objects in our Fermi sample. This is multiplied by the observational PDF to derive the final PDF for each Fermi-detected BL Lac object. If, for example, only $z_{m}$ in and $z_{m} a x$ constraints were available for a given source, its final PDF would follow the prior $d N / d z$ between these limits. As noted below, the prior has only a mild effect on the LF. For each source, then, the PDF is obtained as

$$
\operatorname{PDF}(z)=\frac{d N}{d z} \cdot \prod_{i}^{n} C_{i}(z),
$$



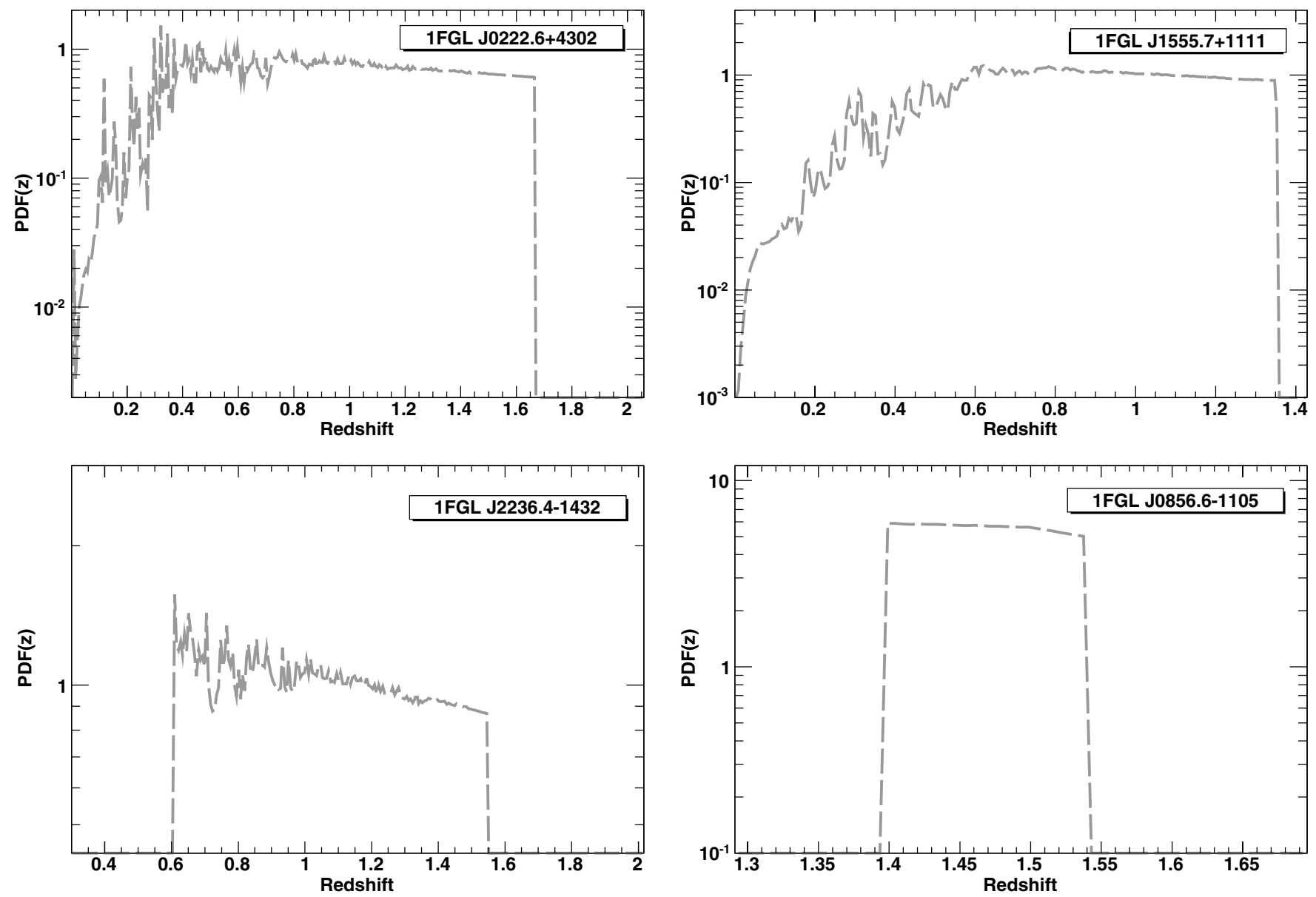

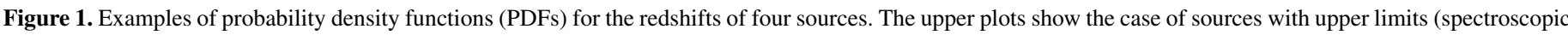

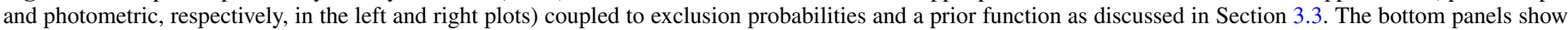

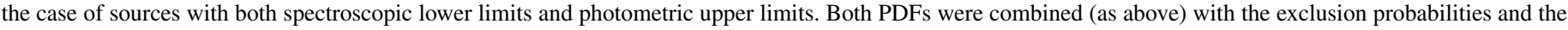
prior function.

where the $C_{i}(z)$ are the redshift constraints available for that source. Sample PDFs are shown in Figure 1.

Drawing possible redshifts from these final PDFs for each source, we compute the sample LF as described above and then use this to predict the observed $d N / d z$ using Equation (6), which represents the redshift distribution expected if all sources could have spectroscopic redshift measurements. In general, this will differ from the initial assumed prior. We replace the prior with this predicted $d N / d z$ and iterate to convergence. Since the $d N / d z$ distribution is rather flat in the range of $0.02<z<2$, we find that the initially assumed prior has very little effect. In practice we find robust convergence to the same final LF for an initial prior $d N / d z \propto z^{-t}$ with $-0.3<t<0.6$. In all cases the derived distribution shows a clear drop in the number of observed BL Lac objects at $z>2$ (see below). However, as this may be an important evolutionary effect that we wish to measure without bias, we conservatively assume a $d N / d z \propto z^{-t}$ prior extending to all $z$ allowed by the constraints. We adopt a computation with an initial $t=0.2$ prior, which is shown in the upper left panel of Figure 2.

\subsection{Summary of the Analysis Chain}

We use a Monte Carlo approach in order to derive the LF and its uncertainty. The steps of the analysis are as follows.
1. An initial prior function (see Section 3.3) is chosen to approximate the $d N / d z$ distribution of the Fermi BL Lac objects.

2. We then create 1000 samples of 206 BL Lac objects whose redshifts are extracted at random from the PDF of each source. The $206 \mathrm{BL} \mathrm{Lac}$ objects ${ }^{15}$ are drawn with replacement from the objects reported in the Appendix.

3. We use the ML method described in Section 3.1 with one of the parameterizations in Section 3.2 to derive the best-fit LF. This is done independently for each Monte Carlo. The final LF is built as the average of the Monte Carlo LFs, and its uncertainty takes into account the spread of all the Monte Carlo LFs. This allows us to quantify naturally the uncertainty in the LF due to the sample size and the spread in the redshift measurements. The LF is used to predict the observed $d N / d z$ through Equation (5).

4. The $d N / d z$ is compared to the prior function used at step 1: If the two functions are different, ${ }^{16}$ then a new prior function based on the latest $d N / d z$ (step 3 ) is created and substituted to the one of step 1 .

15 Including or excluding the five BL Lac objects without redshift information does not change the result of our analysis. When those objects are included, their redshifts are randomly extracted from the prior function.

16 A chi-square fit in the $0.02<z<2$ redshift interval is used to assess the compatibility between the prior function and the $d N / d z$. 

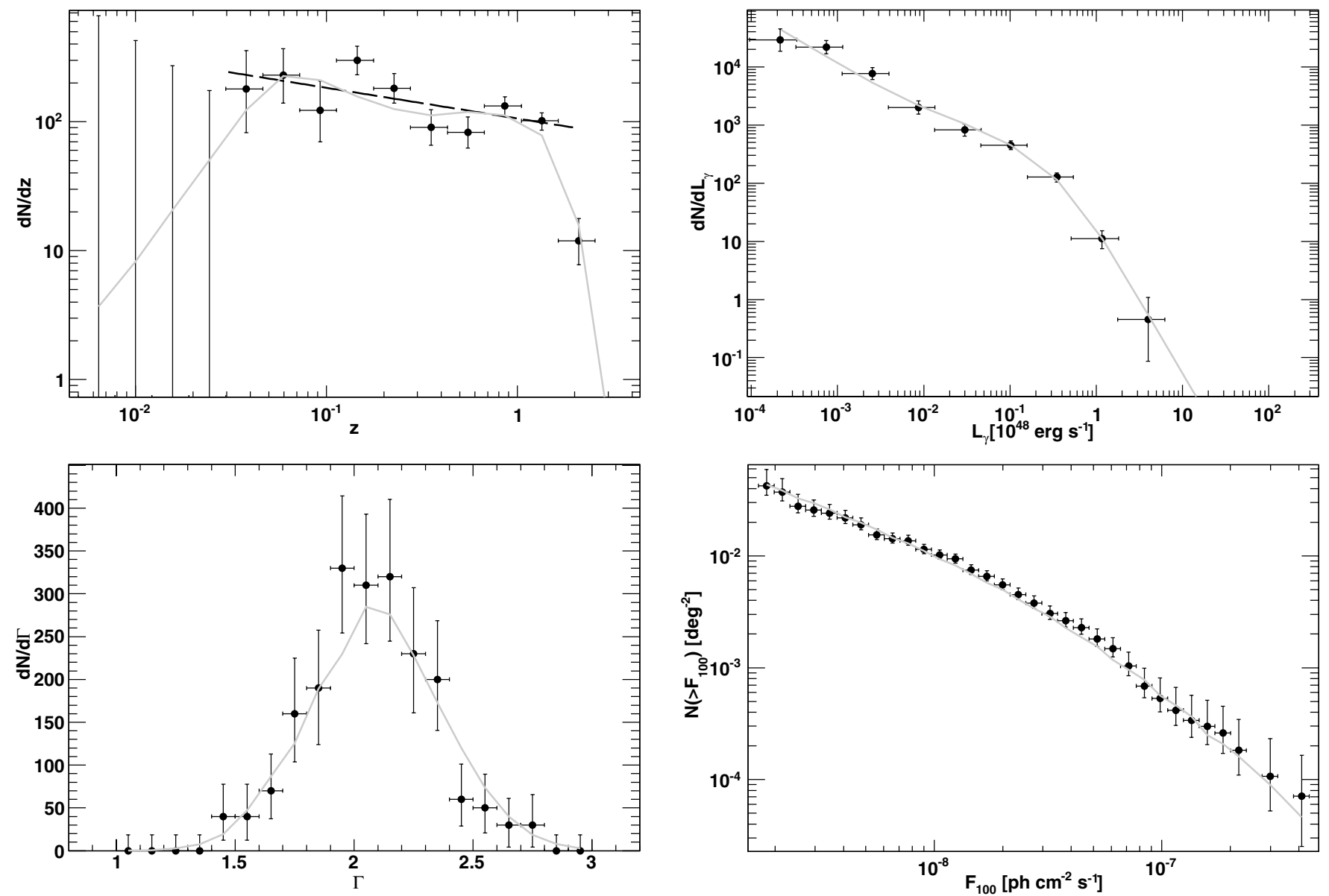

Figure 2. Observed redshift (upper left), luminosity (upper right), photon index (lower left), and source count (lower right) distributions of LAT BL Lac objects. The continuous solid line is the best-fit PLE model convolved with the selection effects of Fermi. The error bars reflect the statistical uncertainty including (for the upper plots) the uncertainty in the sources' redshifts. Error bars compatible with zero are $1 \sigma$ upper limits for the case of observing zero events in a given bin (see Gehrels 1986). The dashed line in the redshift distribution shows one of the prior functions used in Section 3.3.

5. Steps 1-4 are repeated until the prior and the predicted $d N / d z$ are compatible with each other.

We note that a change in the prior function causes a change in the redshift PDFs of all sources, and thus new PDFs have to be created and the entire analysis (steps 2-4) has to be repeated.

\section{RESULTS}

In this section we present results on the best-fitting LF models. Particular attention is given to whether adding the $\beta$ and $\tau$ parameters (representing, respectively, the luminositydependent photon index and a luminosity-dependent speed of evolution; see Equations (11) and (14)) significantly improves the quality of the fit.

\subsection{Density and Luminosity Evolution}

Table 2 reports the results of the best fits using a PDE or a PLE parameterization, including cases for which $\beta$ and $\tau$ are allowed to vary. Both the PLE and PDE LFs provide adequate representations of the Fermi data when $\beta$ and $\tau$ are allowed to vary (see $\mathrm{PLE}_{3}$ and $\mathrm{PDE}_{3}$ models in Table 2). In all cases the PLE model provides a better representation of the Fermi data than the PDE model as indicated by the value of the loglikelihood ( $S$ in Equation (4)). As shown in Figure 2, the best-fit

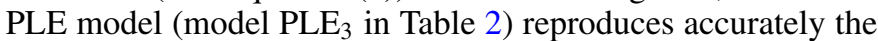
distribution in luminosity, redshift, photon index, and source counts of the Fermi blazars. The model $\mathrm{PLE}_{3}$ provides the best representation of the LF of BL Lac objects.

The improvement in the $\log$-likelihood when $\beta$ and $\tau$ are allowed to vary can be used to quantify the improvement of the fit with the standard formula TS $=-2\left(\ln L_{0}-\ln L_{1}\right)$, where $L_{1}$ is the hypothesis tested against the null one $\left(L_{0}\right)$ and TS is the likelihood TS. We find that allowing the parameter $\beta$ to vary produces an improvement in the fit of TS $>10$ (see Table 2), which corresponds to $>3 \sigma$ for the case of one additional degree of freedom. The $\tau$ parameter that governs the speed of the evolution as a function of luminosity produces an improvement in the fit of TS $=52(\sim 7.2 \sigma)$ for the PLE and TS $=12(\sim 3.4 \sigma)$ for the PDE model.

If we take the luminosities of $10^{45}, 10^{46}$, and $10^{47} \mathrm{erg} \mathrm{s}^{-1}$ as reference luminosities for the Fermi populations of HSPs, ISPs, and LSPs, we then find that the redshift peaks of the luminosity evolution are $z_{c}=0.5,0.8$, and 1.2 , respectively, for these three luminosities. The ML value of the speed of the evolution (parameter $k_{d}$ ) also changes from 4.7 to 5.8 and 7.0, respectively. It thus seems clear that the evolution depends on the luminosity class.

\subsection{Luminosity-dependent Density Evolution}

Given the clear luminosity dependence of the evolution found in the previous section, we try to fit the LDDE model of Section 3.2. This model has two additional parameters with 
Best-Fit Parameters of the Pure Luminosity and Pure Density Evolution LFs

\begin{tabular}{|c|c|c|c|c|c|c|c|c|c|c|c|}
\hline Model & $A^{\mathrm{a}}$ & $\gamma_{1}$ & $L_{*}{ }^{\mathrm{b}}$ & $\gamma_{2}$ & $k$ & $\tau$ & $\xi$ & $\mu^{*}$ & $\beta$ & $\sigma$ & $-2 \ln L^{\mathrm{c}}$ \\
\hline $\mathrm{PLE}_{1}$ & $7.29_{-7.13}^{+31.80} \times 10^{3}$ & $1.26_{-0.20}^{+0.08}$ & $1.42_{-0.94}^{+89.33} \times 10^{-2}$ & $1.31_{-0.09}^{+1.78}$ & $4.87_{-5.39}^{+0.78}$ & 0 & $-0.48_{-0.08}^{+3.48}$ & $2.15_{-0.03}^{+0.03}$ & 0 & $0.27_{-0.02}^{+0.02}$ & -690.1 \\
\hline $\mathrm{PLE}_{2}$ & $2.89_{-2.70}^{+30.91} \times 10^{3}$ & $1.22_{-0.42}^{+0.09}$ & $2.16_{-1.67}^{+73.16} \times 10^{-2}$ & $1.37_{-0.14}^{+2.10}$ & $4.61_{-5.13}^{+0.75}$ & 0 & $-0.48_{-0.10}^{+3.48}$ & $2.12_{-0.03}^{+0.03}$ & $6.48_{-2.09}^{+2.28} \times 10^{-2}$ & $0.26_{-0.02}^{+0.02}$ & -699.9 \\
\hline $\mathrm{PLE}_{3}$ & $9.68_{-4.75}^{+6.88} \times 10^{2}$ & $1.47_{-0.12}^{+0.14}$ & $4.48_{-1.20}^{+2.32} \times 10^{-2}$ & $4.45_{-0.93}^{+1.08}$ & $5.89_{-0.95}^{+0.99}$ & $1.18_{-0.22}^{+0.16}$ & $-0.31_{-0.06}^{+0.05}$ & $2.11_{-0.03}^{+0.03}$ & $6.47_{-2.40}^{+2.23} \times 10^{-2}$ & $0.26_{-0.02}^{+0.03}$ & -752.1 \\
\hline $\mathrm{PLE}_{n o-z}$ & $9.12_{-0.60}^{+0.90} \times 10^{5}$ & $2.07 \pm 0.52$ & $0.12 \pm 0.22$ & $0.77 \pm 0.67$ & $8.60 \pm 1.07$ & $1.41 \pm 0.33$ & $-0.17 \pm 0.04$ & $2.19 \pm 0.04$ & $0.16 \pm 0.04$ & $0.30 \pm 0.04$ & $\cdots$ \\
\hline $\mathrm{PDE}_{1}$ & $78.53_{-73.82}^{+906.10}$ & $1.32_{-0.10}^{+18.68}$ & $0.58_{-0.47}^{+3.01}$ & $1.25_{-0.08}^{+0.09}$ & $11.47_{-1.94}^{+1.44}$ & 0 & $-0.21_{-0.04}^{+0.02}$ & $2.15_{-0.03}^{+0.03}$ & 0 & $0.27_{-0.02}^{+0.02}$ & -695.8 \\
\hline $\mathrm{PDE}_{2}$ & $62.22_{-55.53}^{+989.87}$ & $1.32_{-0.10}^{+18.68}$ & $1.10_{-1.01}^{+2.34}$ & $1.24_{-0.07}^{+0.07}$ & $10.72_{-2.23}^{+1.50}$ & 0 & $-0.24_{-0.06}^{+0.03}$ & $2.12_{-0.03}^{+0.03}$ & $6.33_{-2.00}^{+2.31} \times 10^{-2}$ & $0.26_{-0.02}^{+0.03}$ & -711.9 \\
\hline $\mathrm{PDE}_{3}$ & $18.78_{-14.69}^{+65.86}$ & $3.43_{-0.42}^{+0.78}$ & $0.38_{-0.17}^{+0.46}$ & $1.56_{-0.12}^{+0.16}$ & $16.69_{-2.77}^{+3.52}$ & $3.23_{-0.79}^{+0.85}$ & $-0.11_{-0.02}^{+0.02}$ & $2.10_{-0.03}^{+0.03}$ & $6.45_{-2.31}^{+2.31} \times 10^{-2}$ & $0.26_{-0.03}^{+0.02}$ & -724.8 \\
\hline
\end{tabular}

Notes. Parameters without an error estimate were kept fixed during the fit. Parameter values were computed as the median of all the best-fit parameters to the Monte Carlo sample, while the uncertainties represent the $68 \%$ containment regions around the median value.

${ }^{a}$ In units of $10^{-13} \mathrm{Mpc}^{-3} \mathrm{erg}^{-1} \mathrm{~s}$.

${ }^{\mathrm{b}}$ In units of $10^{48} \mathrm{erg} \mathrm{s}^{-1}$

${ }^{c}$ Value of the $-2 \times \log$-likelihood when the function is minimized. 

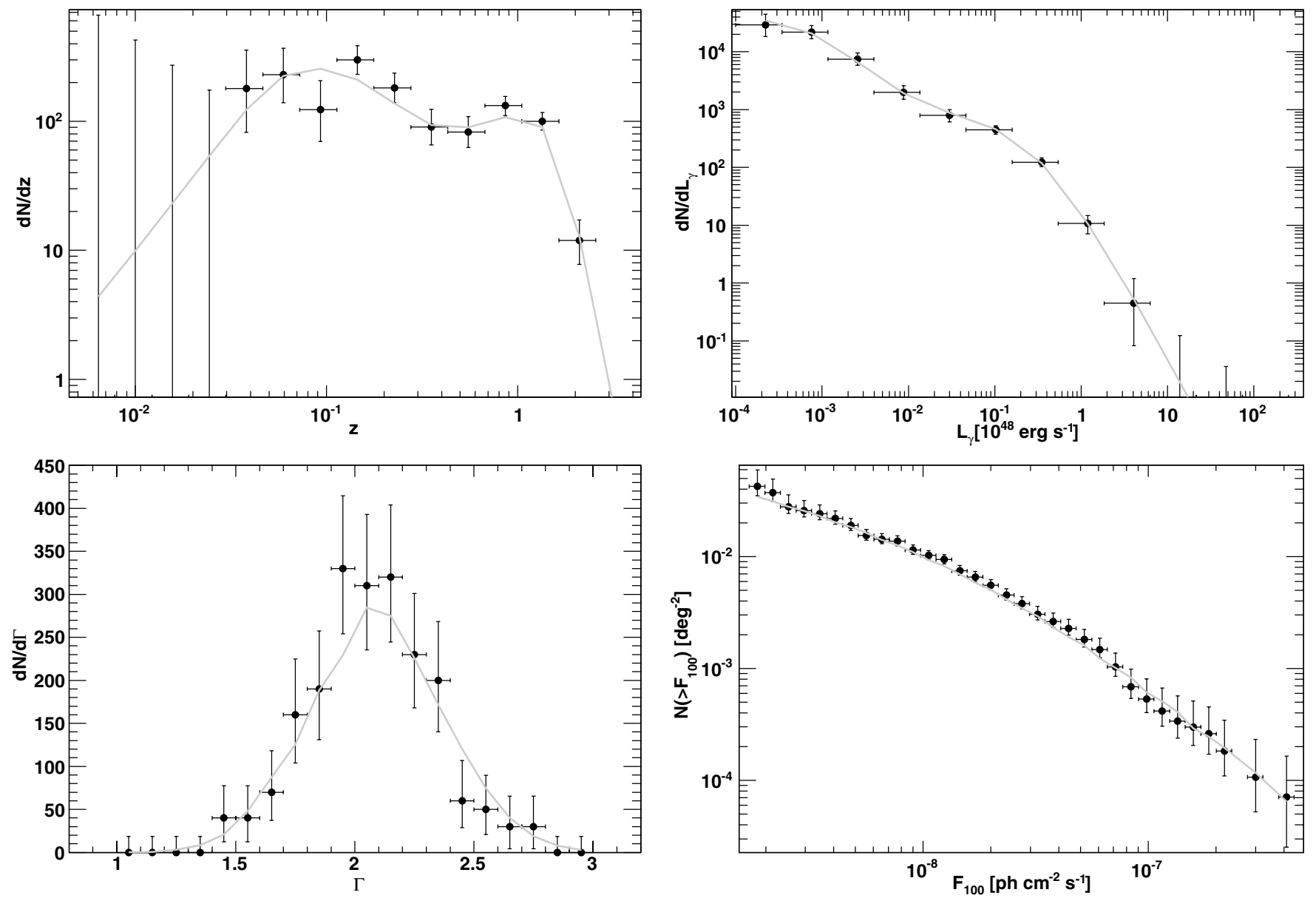

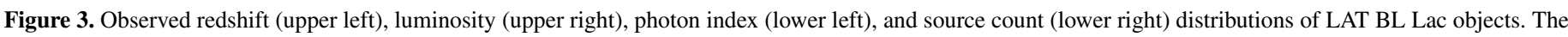

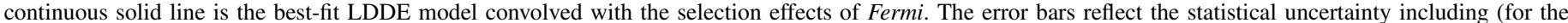

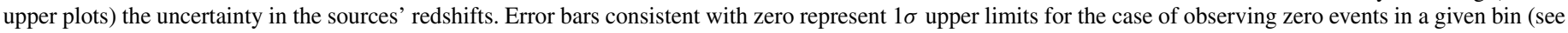
Gehrels 1986).

respect to the PLE and PDE models. The fit with $\tau=0$ (all luminosity classes evolve in the same way) already provides a representation of the data, which is as good as the best-fit PLE model (see Table 3). If we allow $\tau$ to vary, the fit improves further with respect to the baseline $\mathrm{LDDE}_{1}$ model $(\mathrm{TS}=30$, i.e., $\sim 5.5 \sigma)$. Figure 3 shows how the $\mathrm{LDDE}_{3}$ model reproduces the observed distributions.

The improvement of the $\mathrm{LDDE}_{2}$ model with respect to the $\mathrm{PLE}_{3}$ model can be quantified using the Akaike information criterion (AIC; Akaike 1974; Wall \& Jenkins 2012). For each model, one can define the quantity $\mathrm{AIC}_{i}=2 n_{\text {par }}-2 \ln L$, where $n_{\text {par }}$ is the number of free parameters and $-2 \ln L$ is twice the log-likelihood value as reported in Tables 2 and 3. The relative likelihood of a model with respect to another model can be evaluated as $p=e^{0.5\left(\mathrm{AIC}_{\min }-\mathrm{AIC}_{i}\right)}$, where $\mathrm{AIC}_{\text {min }}$ comes from the model providing the minimal AIC value. According to this test, the $\mathrm{PLE}_{3}$ model has a relative likelihood with respect to the $\mathrm{LDDE}_{2}$ model of $\sim 0.0024$. Thus, the model $\mathrm{LDDE}_{2}$ whose parameters are reported in Table 3 fits the Fermi data better $(\sim 3 \sigma)$ than the $\mathrm{PLE}_{3}$ model.

In this representation, low-luminosity $\left(L_{\gamma}=10^{44} \mathrm{erg} \mathrm{s}^{-1}\right)$ sources are found to evolve negatively $(p 1=-7.6)$. On the other hand, high-luminosity $\left(L_{\gamma}=10^{47} \mathrm{erg} \mathrm{s}^{-1}\right)$ sources are found to evolve positively $(p 1=7.1)$. Both evolutionary trends are also correctly represented in the best-fit PLE model $\left(\mathrm{PLE}_{3}\right.$ in Table 2), but the LDDE model provides a slightly better representation of the data. The different evolution of

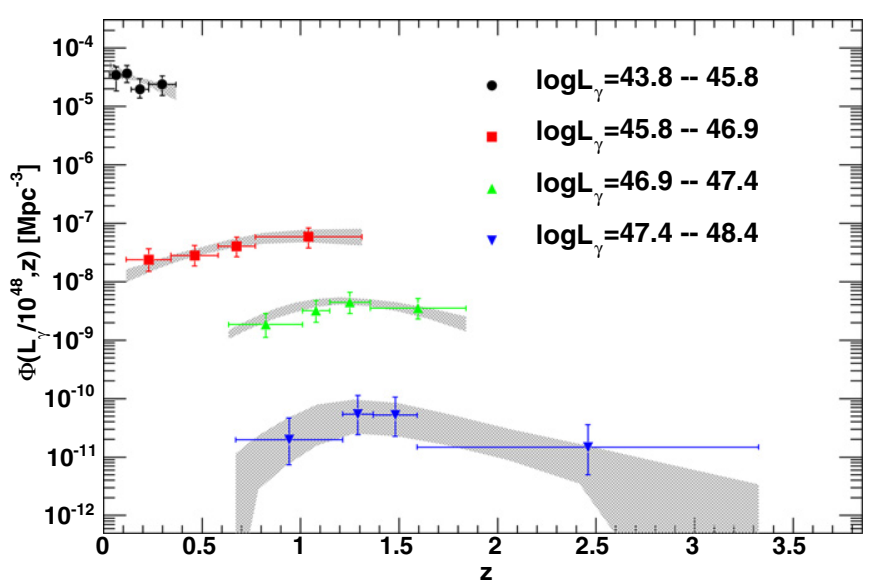

Figure 4. Growth and evolution of BL Lac objects, separated by luminosity class. The gray bands represent $68 \%$ confidence regions around the bestfitting LDDE LF model (for each Monte Carlo sample). Both data points and band errors include uncertainties for the source redshifts as well as statistical uncertainty. All but the least luminous class have a redshift peak near $z \approx 1.5$; the lowest luminosity BL Lac objects increase toward $z=0$.

(A color version of this figure is available in the online journal.)

low-luminosity and high-luminosity sources can be readily appreciated in Figure 4, which shows the space density of different luminosity classes of BL Lac objects as a function of redshift. This figure was created by taking into account the 
Table 3

Best-Fit Parameters of the LDDE LFs

\begin{tabular}{lcccccccccccccc}
\hline \hline Model & $A^{\mathrm{a}}$ & $\gamma_{1}$ & $L_{*}{ }^{\mathrm{b}}$ & $\gamma_{2}$ & $z_{c}^{*}$ & $p 1^{*}$ & $\tau$ & $p 2$ & $\alpha$ & $\mu^{*}$ & $\beta$ & $-2 \mathrm{lnL}$ \\
\hline $\mathrm{LDDE}_{1}$ & $9.20_{-8.77}^{+20.60} \times 10^{2}$ & $1.12_{-0.16}^{+0.13}$ & $2.43_{-1.30}^{+2.25}$ & $3.71_{-2.39}^{+16.29}$ & $1.67_{-0.10}^{+0.14}$ & $4.50_{-0.61}^{+0.75}$ & 0.0 & $-12.88_{-2.12}^{+3.66}$ & $4.46_{-5.24}^{+6.47} \times 10^{-2}$ & $2.12_{-0.03}^{+0.03}$ & $6.04_{-2.02}^{+2.15} \times 10^{-2}$ & $0.26_{-0.02}^{+0.02}$ & -734.1 \\
$\mathrm{LDDE}_{2}$ & $3.39_{-2.13}^{+7.44} \times 10^{4}$ & $0.27_{-0.46}^{+0.26}$ & $0.28_{-0.21}^{+0.43}$ & $1.86_{-0.48}^{+0.86}$ & $1.34_{-0.27}^{+0.22}$ & $2.24_{-1.07}^{+1.25}$ & $4.92_{-2.12}^{+1.45}$ & $-7.37_{-5.43}^{+2.95}$ & $4.53_{-6.52}^{+4.98} \times 10^{-2}$ & $2.10_{-0.03}^{+0.03}$ & $6.46_{-2.07}^{+2.34} \times 10^{-2}$ & $0.26_{-0.02}^{+0.02}$ & -764.6 \\
\hline $\mathrm{LDDE}_{\text {noProb }}$ & $1.04_{-0.74}^{+14.90} \times 10^{4}$ & $0.58_{-0.75}^{+0.18}$ & $0.50_{-0.47}^{+0.75}$ & $1.99_{-0.70}^{+1.70}$ & $1.18_{-0.27}^{+0.38}$ & $2.30_{-1.17}^{+2.11}$ & $4.62_{-1.73}^{+5.38}$ & $-4.30_{-4.50}^{+2.07}$ & $8.62_{-13.30}^{+5.55} \times 10^{-2}$ & $2.11_{-0.03}^{+0.03}$ & $6.64_{-2.05}^{+1.84} \times 10^{-2}$ & $0.26_{-0.02}^{+0.02}$ & -985 \\
\hline
\end{tabular}

Notes. Parameters without an error estimate were kept fixed during the fit. Parameter values were computed as the median of all the best-fit parameters to the Monte Carlo sample, while the uncertainties represent the $68 \%$ containment region around the median value.

${ }^{a}$ In unit of $10^{-13} \mathrm{Mpc}^{-3} \mathrm{erg}^{-1} \mathrm{~s}$.

${ }^{\mathrm{b}}$ In unit of $10^{48} \mathrm{erg} \mathrm{s}^{-1}$.

${ }^{c}$ Value of the $-2 \times \log$-likelihood when the function is minimized. 


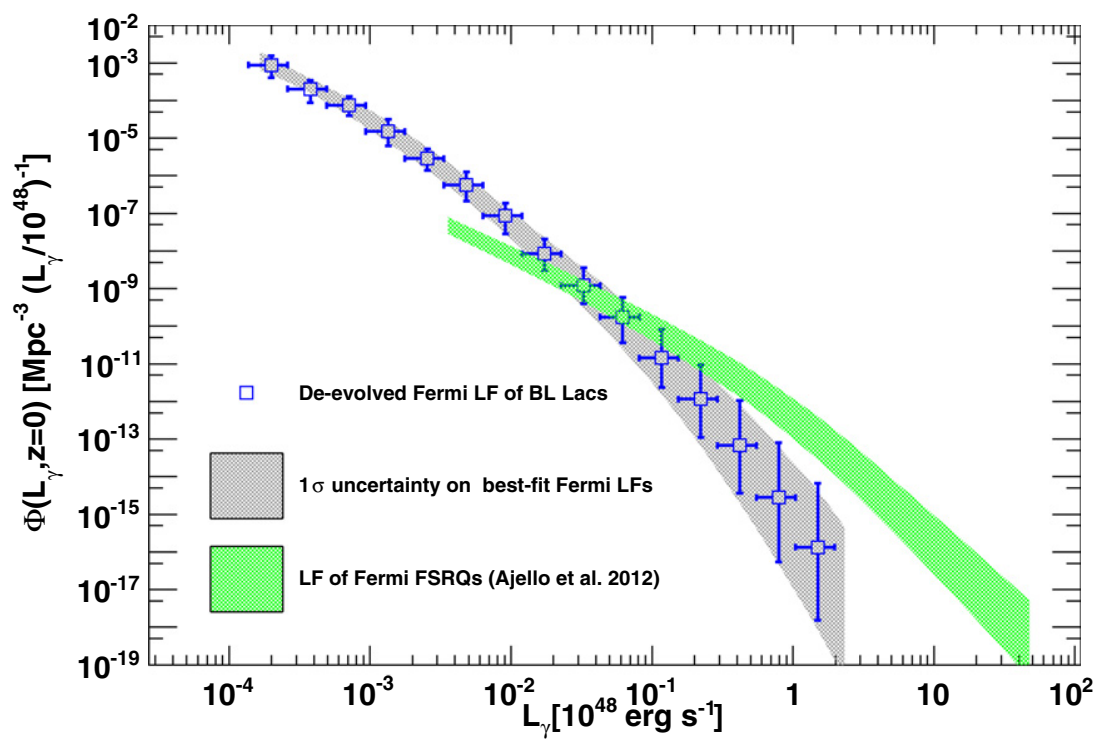

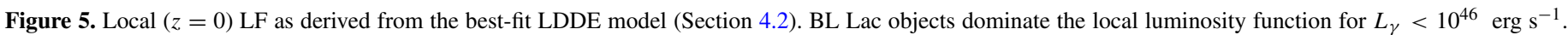
The gray band represents the confidence region enclosing $68 \%$ of the realizations of the best-fit LF to the Monte Carlo samples.

(A color version of this figure is available in the online journal.)

dispersion in both redshift and luminosity introduced by the uncertainty in the redshift of many of our BL Lac objects. A noteworthy fact is that the least luminous BL Lac objects are $10^{3}$ times more numerous than the least luminous FSRQs detected by Fermi (see Figure 4 in Ajello et al. 2012). The data points were deconvolved with the method described in Section 3.1 (see Equation (9)), while the LF is displayed as the region enclosing $68 \%$ of all the best-fit LDDE models to the 1000 Monte Carlo samples.

The local LF is the LF at redshift zero. For an evolving population, the local LF is obtained by de-evolving the luminosities (or the densities) according to the best-fit model. We follow two approaches to derive the local LF. First, we de-evolve the luminosities using the $1 / V_{\text {MAX }}$ method of Schmidt (1968) weighting the maximum volume ( $\left.V_{\text {MAX }}\right)$ by the density evolution implied (for a given source luminosity) by our best-fit LDDE model. Following Della Ceca et al. (2008) and Ajello et al. (2012), the maximum allowed volume for a given source is defined as

$$
V_{\mathrm{MAX}}=\int_{z_{\min }}^{z_{\max }} \Omega\left(L_{i}, z, \Gamma\right) \frac{e\left(z, L_{i}\right)}{e\left(z_{\min }, L_{i}\right)} \frac{d V}{d z} d z,
$$

where $L_{i}$ is the source luminosity, $\Omega\left(L_{i}, z, \Gamma\right)$ is the sky coverage, $z_{\max }$ is the redshift above which the source drops out of the survey, and $e\left(z, L_{i}\right)$ is the evolution term of Equation (13) normalized (through $\left.e\left(z_{\min }, L_{i}\right)\right)$ at the redshift $z_{\min }$ to which the $\mathrm{LF}$ is to be de-evolved. The LF de-evolved at $z_{\min }\left(z_{\min }=0\right.$ in this case) is built using the standard $1 / V_{\text {MAX method (Schmidt }}$ 1968). This is reported (data points) in Figure 5. To estimate the uncertainties that different methods might introduce in the local LF we also extrapolated to $z=0$ from the best-fit LDDE models to all the Monte Carlo samples to measure the $68 \%$ range for the local LF. This is shown in Figure 5 as a gray band. It is apparent that the two methods give consistent results.

The local LF is found to have a rather steep power-law $\left(d N / d L \propto L^{-3.5}\right)$ down to luminosities of $10^{46} \mathrm{erg} \mathrm{s}^{-1}$, flattening $\left(d N / d L \propto L^{-2.0}\right)$ below this value. Because of their steeper local LF and their lower luminosity, BL Lac objects reach higher densities than FSRQs (whose local LF is shown for comparison in Figure 5). Figure 6 shows the evolution of the luminosity density of BL Lac objects compared to that of FSRQs. With their larger luminosity, FSRQs dominate at all redshifts $z>0.3$. Yet the extreme growth in BL Lac numbers at low $z$ allows them to produce $>10^{45} \mathrm{erg} \mathrm{yr}^{-1} \mathrm{Mpc}^{-3}$, or $\sim 90 \%$ of the local luminosity density.

\subsection{The Effect of Neglecting Redshift Constraints}

Neglecting redshift constraints and relying only on spectroscopic redshifts reduces the completeness of our sample to only $\sim 48 \%$. As we show in the following section, this has dramatic effects on the reliability of the LF.

The main reason is that the distribution of spectroscopic redshifts poorly approximates the redshift distribution of $\mathrm{BL}$ Lac objects inferred using all the redshift constraints presented in Section 3.3. This can clearly be seen in Figure 7, which compares the BL Lac redshift distribution taking all constraints into account compared to known BL Lac redshift distributions based solely on spectroscopic redshifts. These latter are biased to find low redshift BL Lac objects, while it is clear from recent works (Rau et al. 2012; Shaw et al. 2013a, 2013b; Furniss et al. 2013) that there is a relevant population of BL Lac objects at intermediate $(z \approx 0.5-1.5)$ redshift. This is not a spurious effect caused by any of the techniques presented in Section 3.3, but evidence that comes from all of them. In order to test this, we removed the exclusion probabilities from the used constraints and re-derived the LF. The exclusion probability is available for all but $5 \mathrm{BL}$ Lac objects without redshift and on average constrains a given object at $z \gtrsim 0.3-0.5$. If wrong, it might artificially push the average redshift of BL Lac objects to higher values. We find this is not the case. Indeed, even when removing the exclusion probabilities the redshift distribution of BL Lac objects still shows an increase at $z>0.5$, which is this time mostly due to the redshift lower limits. Moreover as reported in Table 3, the LF derived from discarding only the exclusion probabilities (see model $\mathrm{LDDE}_{\text {noProb }}$ ) is still in agreement with the best-fitting model $\left(\mathrm{LDDE}_{2}\right)$ that relies on all constraints.

As expected from the above discussion if we neglect all redshift constraints and rely only on the 103 BL Lac objects with spectroscopic redshifts, the best-fit LF (reported as model 


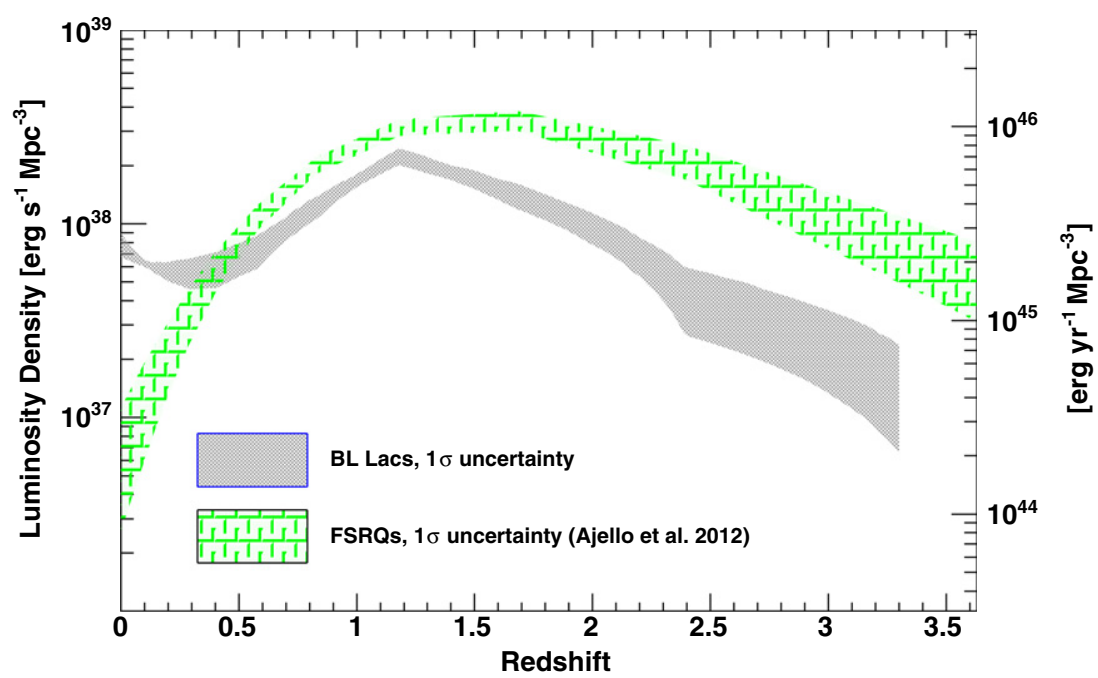

Figure 6. Luminosity density as a function of redshift produced by the Fermi BL Lac objects. The gray band represents the confidence region enclosing $68 \%$ of the realizations of the best-fit LF to the Monte Carlo samples.

(A color version of this figure is available in the online journal.)

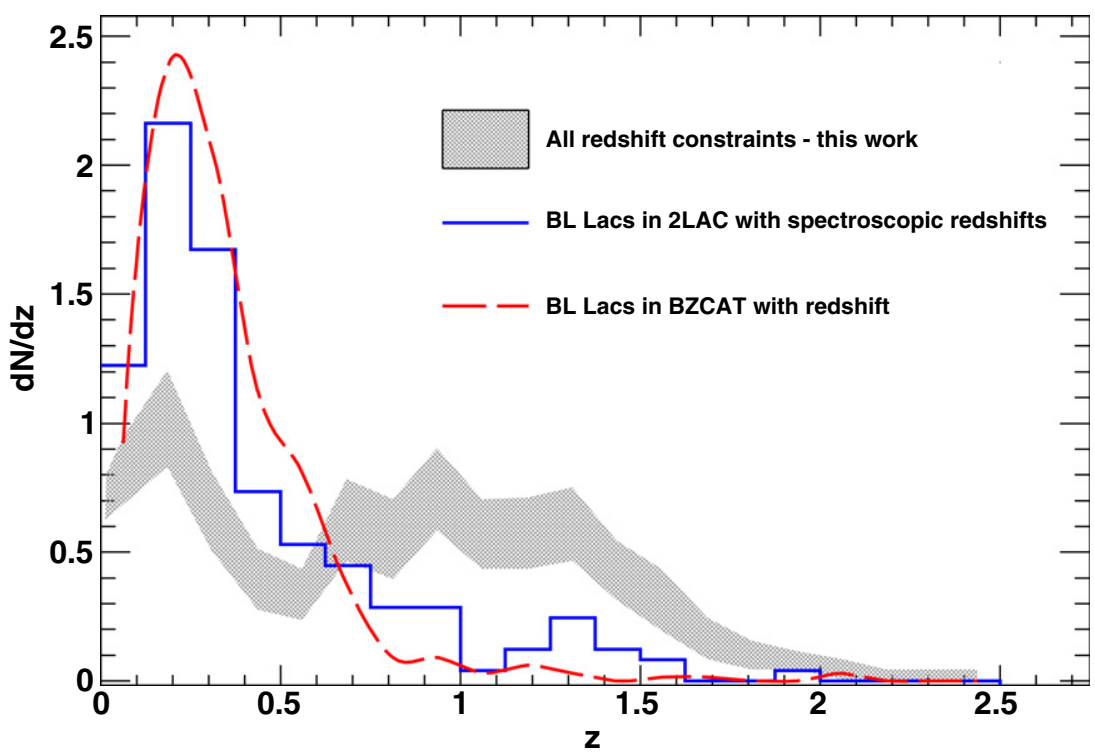

Figure 7. Redshift distribution of Fermi's BL Lac derived using all constraints of Section 3.3 compared to spectroscopic redshift distributions of BL Lac objects in the 2LAC catalog (Ackermann et al. 2011) and the Roma blazar catalog (BZCAT; Massaro et al. 2009). The gray band encloses the 68\% of all realizations of the redshift distribution of the Monte Carlo samples.

(A color version of this figure is available in the online journal.)

$\mathrm{PLE}_{n o-z}$ in Table 2) changes fairly dramatically with respect to the best-fit $\mathrm{LDDE}_{2}$ model. Indeed, instead of showing a change in the evolution with source luminosity, it displays a very mild positive evolution for all luminosity classes. This would lead to a biased estimate of the evolution of BL Lac objects. We thus believe that results based on BL Lac samples with scarce redshift coverage are unreliable.

\subsection{The Intrinsic Luminosity Function of BL Lac Objects}

Beaming is known to alter the shape of the intrinsic LF (e.g., Urry \& Shafer 1984; Urry \& Padovani 1991). In this section we correct for this effect, recovering the intrinsic LF of the Fermi BL Lac objects and their Lorentz and Doppler factor distributions. Here we adopt the formalism and symbols already used in Ajello et al. (2012).
The observed $0.1-100 \mathrm{GeV}$ luminosities $L$ defined in the present work are apparent isotropic luminosities (expressed in erg $\mathrm{s}^{-1}$ ). Since the jet material is moving at relativistic speed, the observed Doppler-boosted luminosities are related to the intrinsic values by

$$
L=\delta^{p} \mathscr{L}
$$

where $\mathscr{L}$ is the intrinsic (unbeamed) luminosity and $\delta$ is the kinematic Doppler factor

$$
\delta=\left(\gamma-\sqrt{\gamma^{2}-1} \cos \theta\right)^{-1},
$$

where $\gamma=\left(1-\beta^{2}\right)^{-1 / 2}$ is the Lorentz factor, $\beta=v / c$ is the velocity of the emitting plasma, and $\theta$ is the angle between the line of sight and the jet axis. We will assume that our sources have Lorentz factors $\gamma$ in the range of $\gamma_{a} \leqslant \gamma \leqslant \gamma_{b}$ : then the 


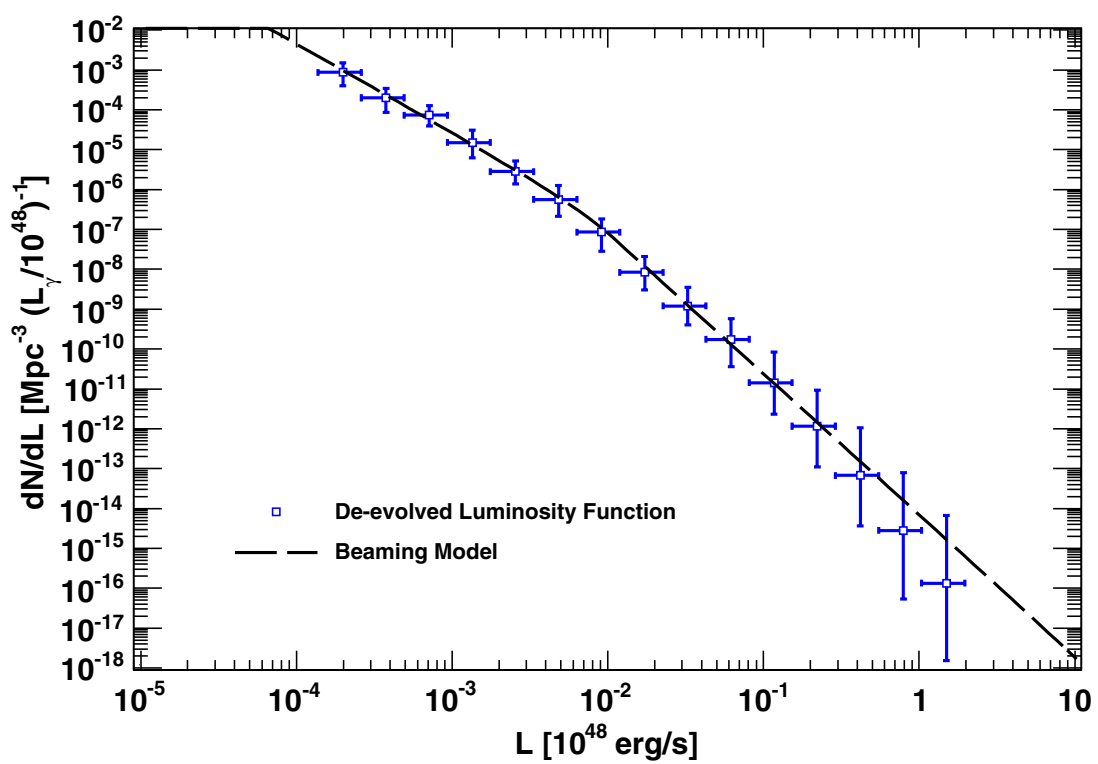

Figure 8. Fermi's LF de-evolved at redshift zero and best-fit beaming model (for $p=4$, dashed line, see text) described in Section 4.4 .

(A color version of this figure is available in the online journal.)

minimum Doppler factor is $\delta_{\min }=\gamma_{b}^{-1}\left(\right.$ when $\left.\theta=90^{\circ}\right)$ and the maximum is $\delta_{\max }=\left(\gamma_{a}+\sqrt{\gamma_{a}^{2}-1}\right)^{-1}$ (when $\left.\theta=0^{\circ}\right)$. We adopt a value of $p=4$, which is appropriate if the observed emission is dominated by the synchrotron self-Compton component of ejected plasma blobs; we also discuss the case of $p=3$, which applies to the case of continuous jet emission.

We define the intrinsic LF as

$$
\Phi(\mathscr{L})=k_{1} \mathscr{L}^{-B},
$$

which is valid in the $\mathscr{L}_{1} \leqslant \mathscr{L} \leqslant \mathscr{L}_{2}$ range. The joint probability of observing a beamed luminosity $L$ and Doppler factor $\delta$ is (see also Lister 2003)

$$
P(L, \delta)=P_{\delta}(\delta) \cdot \Phi(\mathscr{L}) \frac{d \mathscr{L}}{d L},
$$

where $P_{\delta}(\delta)$ is the probability density for the Doppler $\delta$ and $d \mathscr{L} / d L=\delta^{-p}$. Assuming a random distribution for the jet angles (i.e., $P_{\theta}=\sin \theta$ ), this results in

$$
P_{\delta}(\delta)=\int P_{\gamma}(\gamma) P_{\theta}(\theta)\left|\frac{d \theta}{d \delta}\right| d \gamma=\int P_{\gamma}(\gamma) \frac{1}{\gamma \delta^{2} \beta} d \gamma,
$$

since

$$
\left|\frac{d \theta}{d \delta}\right|=\frac{1}{\sin (\theta) \delta^{2} \sqrt{\gamma^{2}-1}}=\frac{1}{\sin (\theta) \delta^{2} \gamma \beta} .
$$

From here it follows that

$$
P_{\delta}(\delta)=\delta^{-2} \int_{f(\delta)}^{\gamma_{b}} \frac{P_{\gamma}(\gamma)}{\sqrt{\gamma^{2}-1}} d \gamma
$$

where $P_{\gamma}(\gamma)$ is the probability density for $\gamma$, and the lower limit of integration $f(\delta)$ depends on the Doppler factor value and is reported in Equation (A6) in Lister (2003). Integrating over $\delta$ yields the observed LF of the Doppler beamed BL Lac objects:

$$
\Phi(L)=k_{1} L^{-B} \int_{\delta_{1}(L)}^{\delta_{2}(L)} P_{\delta}(\delta) \delta^{p(B-1)} d \delta,
$$

where, as in Cara \& Lister (2008), the limits of integration are

$$
\begin{aligned}
& \delta_{1}(L)=\min \left\{\delta_{\max }, \max \left(\delta_{\min },\left(L / \mathscr{L}_{2}\right)^{1 / p}\right)\right\}, \\
& \delta_{2}(L)=\max \left\{\delta_{\min }, \min \left(\delta_{\max },\left(L / \mathscr{L}_{1}\right)^{1 / p}\right)\right\} .
\end{aligned}
$$

In this way, by fitting Equation (30) to the Fermi Dopplerboosted LF, it is possible to determine the parameters of the intrinsic LF and the Lorentz-factor distribution.

We assume that the probability density distribution for $\gamma$ is a power-law of the form

$$
P_{\gamma}(\gamma)=C \gamma^{k},
$$

where $\mathrm{C}$ is a normalization constant and the function is valid for $\gamma_{a} \leqslant \gamma \leqslant \gamma_{b}$. We set the largest intrinsic luminosity $\mathcal{L}_{2}=10^{4} \mathcal{L}_{1}$, but this choice has hardly any impact on the results. Fits with parameters similar to those of FSRQs $(p=4$, $\gamma_{a}=5, \gamma_{b}=40$, and $\left.\mathcal{L}_{1}=10^{40} \mathrm{erg} \mathrm{s}^{-1}\right)$ are ruled out $\left(\chi^{2} / \mathrm{dof}\right.$ $>2.5$ ). In order to obtain acceptable fits we find that $\mathcal{L}_{1}$ has to be set to $\leqslant 10^{40} \mathrm{erg} \mathrm{s}^{-1}$ or $\leqslant 10^{38} \mathrm{erg} \mathrm{s}^{-1}$ for the $p=3$ and $p=4$ cases, respectively. Moreover, in agreement with the observation of BL Lac objects in radio (e.g., Lähteenmäki \& Valtaoja 2003; Lister et al. 2009; Savolainen et al. 2010) we set $\gamma_{a}=2$, which is lower than the minimum value used (and found) for FSRQs (see, e.g., Ajello et al. 2012). In order to allow for a population of highly beamed BL Lac objects we set $\gamma_{b}=90$.

The free parameters of the problem are the normalization $\left(k_{1}\right)$, the slope $(B)$ of the intrinsic LF, and the slope $k$ of the Lorentz factor distribution. We have fitted Equation (30) to the Fermi LF de-evolved at redshift zero derived in Section 4.2. Figure 8 shows how the best-fit beaming model reproduces the local LF of BL Lac objects measured by Fermi. For the $p=4$ case we can use the fit values to derive an intrinsic LF slope of $B=3.30 \pm 0.30$ and a Lorentz-factor distribution index of $k=-2.32 \pm 0.51$. The parameters for the $p=3$ case are similar. Our distribution of Lorentz factors are somewhat steeper than (but compatible with, within the uncertainties) those found by Lister \& Marscher (1997), who report a slope of $-1.75<k<-1.5$. The fit values are summarized in Table 4 . 


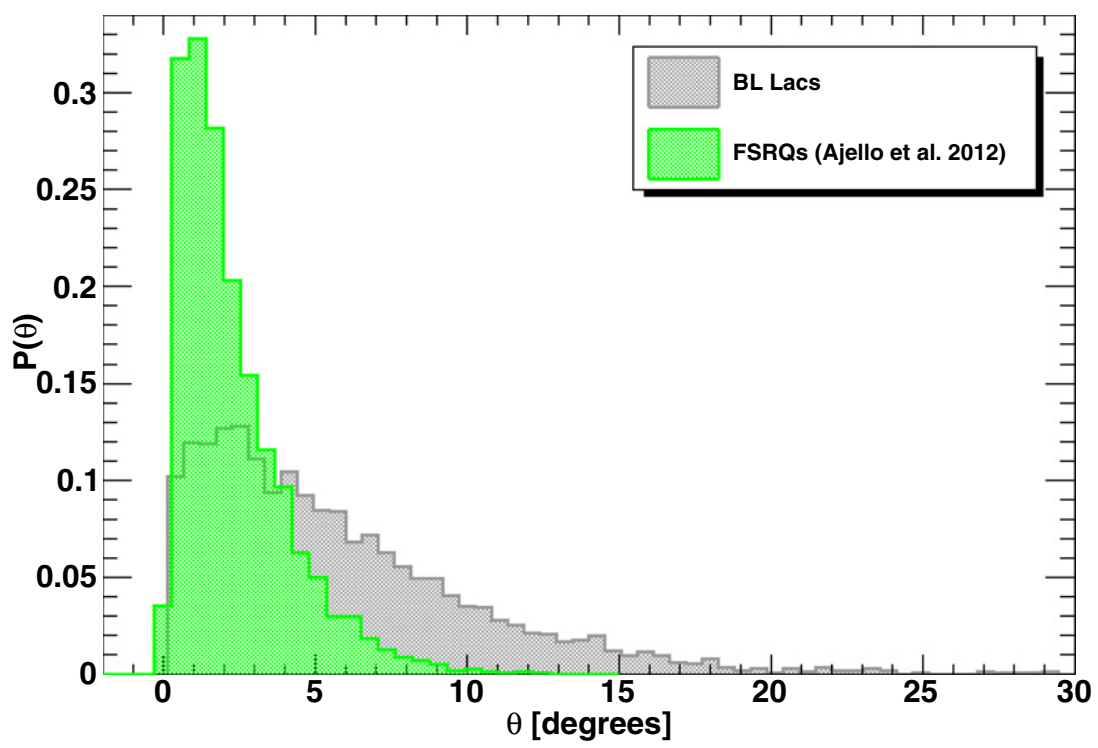

Figure 9. Normalized distributions of viewing angles with respect to the jet axis for Fermi BL Lac objects and FSRQs.

(A color version of this figure is available in the online journal.)

Table 4

Parameters of the Beaming Models Described in the Text

\begin{tabular}{lcc}
\hline \hline Parameter & Value & Value \\
\hline$k$ & $-2.26 \pm 0.20$ & $-2.32 \pm 0.51$ \\
$k_{1}$ & $4.3 \pm 0.5^{\mathrm{a}}$ & $2.7 \pm 0.5^{\mathrm{a}}$ \\
$B$ & $3.96 \pm 0.08$ & $3.30 \pm 0.30$ \\
$\gamma_{a}$ & 2 & 2 \\
$\gamma_{b}$ & 90 & 90 \\
$\mathscr{L}_{1}$ & $10^{40}$ & $10^{38}$ \\
$\mathscr{L}_{2}$ & $10^{44}$ & $10^{42}$ \\
$p$ & 3 & 4 \\
$\chi^{2} /$ dof & 0.3 & 0.21 \\
Average $\gamma$ & $6.1_{-0.8}^{+1.1}$ & $5.8_{-1.6}^{+3.6}$ \\
\hline
\end{tabular}

Notes. Parameters without an error estimate were kept fixed during the fitting stage.

${ }^{a}$ In units of $10^{-27}$.

The Lorentz-factor distributions (for the $p=3$ and $p=4$ cases) imply an average Lorentz factor of $\gamma \approx 6$ for the detected Fermi blazars. This is in agreement with past inferences for radio and X-ray-selected BL Lac objects (see discussion in Urry \& Padovani 1995; Morganti et al. 1995). The average Lorentz factor depends on the value adopted for $\gamma_{a}$ (and to a lesser extent on $\gamma_{b}$ ). Within the errors, the slope $k$ is the same for BL Lac objects and FSRQs $(-2.32 \pm 0.51$ versus $-2.03 \pm 0.70$, respectively). The fact that it is not possible to produce a good fit to the data adopting the same $\gamma_{a}$ for both populations implies that a population of BL Lac objects exists with jets slower than those of FSRQs. This yields a smaller value for the average Lorentz factor $\left(\gamma^{\text {BL Lac }} \approx 6\right.$ versus $\left.\gamma^{\mathrm{FSRQ}} \approx 12\right)$ and shows that BL Lac objects are seen under larger angles $\left(\sim 5^{\circ}\right.$ versus $\sim 2{ }^{\circ}$ for FSRQs, see Figure 9).

Finally, we also tested different parameterizations of the distribution of Lorentz factors (Equation (33)). We used a linear, an exponential, and a Gaussian distribution. None of these models provides an acceptable fit to the data $\left(\chi^{2} /\right.$ dof $\left.>3\right)$. We thus conclude that parameterizing the Lorentz factor distribution with a power-law model (as done also in the literature, e.g., Urry \& Shafer 1984; Cara \& Lister 2008) is a reasonable assumption.

\section{SUBCLASSES OF BL LAC OBJECTS}

Our sample can be subdivided into 96 HSPs, 64 ISPs, and 45 LSPs on the basis of the frequency of the synchrotron peak (see Ackermann et al. 2011). For only six objects there is not enough multiwavelength coverage to accurately define the position of the synchrotron peak. It is thus possible to test whether the different subclasses of BL Lac objects have different evolutions. In particular we are interested in testing the following two scenarios: (1) whether HSPs have a different evolution with respect to ISPs and LSPs, and (2) whether LSPs have a different evolution with respect to HSPs and ISPs. For completeness the best-fit parameters of all the models described in Sections 5.1 and 5.2 are reported in Tables 6 and 7 in Appendix A.2.

\subsection{The Evolution of HSP Objects}

Using the same best-fit models (namely the PLE and LDDE models of Section 3.2) we next examine the HSP objects separately. The LDDE model is slightly preferred to the PLE model ( TS $\approx 12$ ). Both models indicate that the evolution of the HSP is negative, i.e., the density is growing with decreasing redshift.

For the PLE model, the relevant parameters are $k=3.82_{-1.17}^{+1.29}$, $\tau=1.35_{-0.32}^{+0.17}$, and $\gamma=-0.40_{-0.14}^{+0.07}$. For all the HSPs with $L_{\gamma} \leqslant 10^{46} \mathrm{erg} \mathrm{s}^{-1}$ the evolution is negative $z_{c} \leqslant 0$ and $k_{d} \leqslant 0$. The same trend is confirmed by the LDDE model whose relevant parameters are $p 1^{*}=0.48_{-0.48}^{+1.63}$, and $\tau=6.76_{-1.82}^{+2.33}$ (see Equation (20)).

For the class of ISP and LSP objects the LDDE model produces a very small improvement over the PLE model $(\mathrm{TS} \approx 3)$. Both models indicate positive evolution for the ISPs and LSPs considered together. For the PLE, the parameters that govern the evolution are $k=7.86_{-1.86}^{+1.41}, \tau=0.98_{-0.31}^{+0.28}$, and $\xi=-0.25_{-0.08}^{+0.05}$. In this scenario low-luminosity sources are characterized by a slow positive evolution consistent with no evolution.

The different evolutionary behavior of HSPs with respect to all other blazar classes can be appreciated in Figure 10, which 


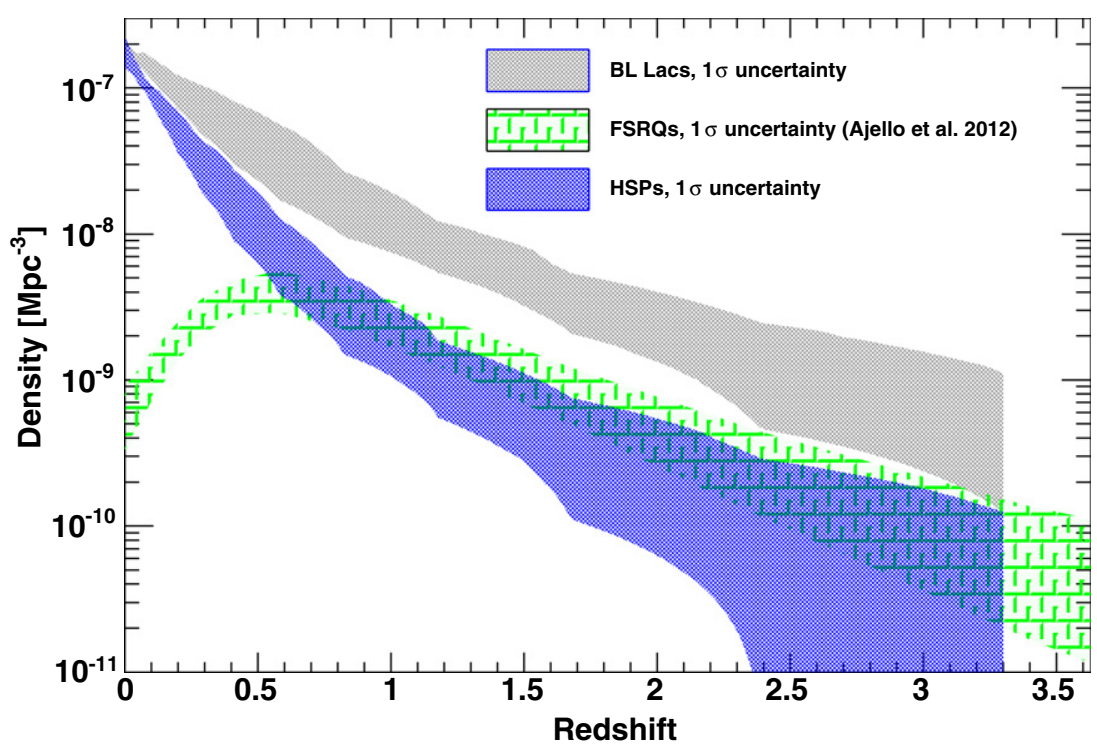

Figure 10. Number density (per unit comoving volume) of BL Lac objects, FSRQs, and HSPs. (A color version of this figure is available in the online journal.)
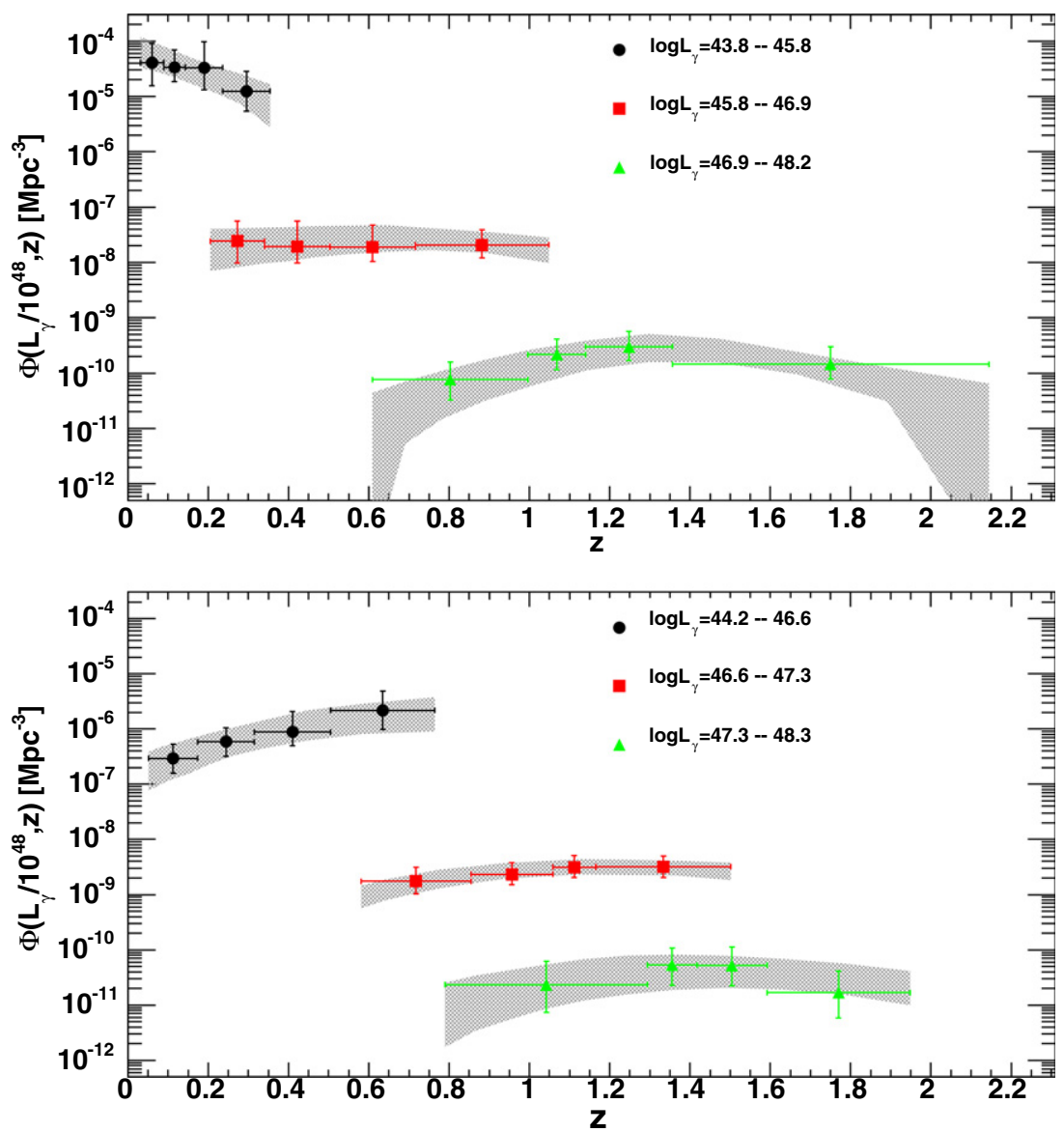

Figure 11. Evolution of different luminosity classes of HSPs (top) and ISPs+LSPs (bottom). Note the different evolutionary behavior (negative for HSPs vs. positive for ISPs+LSPs evolution) of low-luminosity sources.

(A color version of this figure is available in the online journal.)

shows that the dramatic rise in the number density of BL Lac objects at $z \leqslant 1$ is driven almost entirely by the HSP population. The fact that low-luminosity HSP objects are the only ones experiencing negative evolution can also be seen directly in Figure 11.

\subsection{The Evolution of LSP Objects}

LSP objects are the class of BL Lac objects that most closely resemble the FSRQ class. Their synchrotron component peaks at frequencies $<10^{14} \mathrm{~Hz}$ (Ackermann et al. 2011), they can show 
rather large values of the Compton dominance ${ }^{17}$ (Finke 2013), and their average redshift is larger than that of the rest of the BL Lac objects. A number of LSPs might be FSRQs whose jet is aligned along our line-of-sight and whose nonthermal radiation reduces the equivalent width of optical lines. Indeed, Shaw et al. (2013b) find that many BL Lac sources (especially LSPs) are spectrally classified as FSRQ when seen in low states. Since the FSRQ class is known to evolve positively (Ajello et al. 2012), the close connection between FSRQs and high luminosity BL Lac objects might be responsible for the positive evolution detected for the high-luminosity objects in Section 4.2.

The model that best describes the LF of LSP objects is the PLE model. The best-fit evolutionary parameters of the PLE model $\left(k=7.59_{-2.09}^{+1.78}, \tau=1.30_{-0.39}^{+0.26}\right.$, and $\left.\xi=-0.23_{-0.08}^{+0.05}\right)$ imply a strong positive evolution for LSP objects of all luminosities as is the case for FSRQs (see, e.g., Ajello et al. 2012).

The LDDE model ${ }^{18}$ applied to HSPs and ISPs yields $p_{1}^{*}=1.98_{-1.20}^{+1.46}$ and $\tau=6.38_{-1.66}^{+1.58}$. These parameters are in agreement with those of the full sample (reported in Table 3) and imply negative evolution for low-luminosity objects $\left(L_{\gamma} \leqslant 10^{46} \mathrm{erg} \mathrm{s}^{-1}\right)$ and positive evolution for high-luminosity objects $\left(L_{\gamma}>10^{46} \mathrm{erg} \mathrm{s}^{-1}\right)$.

For high-luminosity BL Lac objects $\left(L_{\gamma} \sim 10^{47} \mathrm{erg} \mathrm{s}^{-1}\right)$ both models described above find a positive evolution with $k_{d} \approx 8.9$ (for the PLE model) and $p_{1} \approx 8-9$ (for the LDDE). As such it is apparent that LSPs are not driving the positive evolution of the whole BL Lac sample but that this is a characteristic of all high-luminosity BL Lac objects.

\section{DISCUSSION AND CONCLUSIONS}

In this work we determined the first LF of GeV-detected BL Lac objects. This was made possible by the relatively complete redshift information gleaned from a variety of methods (see, e.g., Rau et al. 2012; Shaw et al. 2013b), leaving only 5 of our 211 BL Lac objects without redshift constraints. Previous BL Lac samples selected at other frequencies contained few objects (often $<50$ ) and typically lacked redshift information for $\geqslant 30 \%$ of the objects (see, e.g., Stickel et al. 1991; Padovani et al. 2007; Marcha \& Caccianiga 2013). Poor redshift completeness renders the LF unreliable (see Section 4.3). Also, our sample contains a substantial number of BL Lac objects from each of the three spectral peak subclasses and covers a large redshift range. As such, this sample stands as the largest and most complete (redshift wise) set of BL Lac objects ever used at any frequency and has allowed a greatly improved characterization of the BL Lac population, beaming, and evolution. The main results of our analysis are discussed below.

\subsection{The Evolution of the BL Lac Luminosity Function}

In the past, BL Lac objects have been found to show a wide range of evolutionary patterns. Rector et al. (2000), Giommi et al. (1999), and Beckmann et al. (2003), whose samples contained large fractions of HSP objects, found the BL Lac objects to evolve negatively; Caccianiga et al. (2002) and Padovani et al. (2007) found that BL Lac objects do not evolve; and recently Marcha \& Caccianiga (2013) reported on a sample with positive evolution. These different results were likely due to limited statistics and inadequate redshift coverage mixed with a selection of different classes of BL Lac objects.

\footnotetext{
17 The Compton dominance is the ratio between the Compton peak luminosity and the synchrotron peak luminosity.

18 The PLE model produces a worse fit $(\mathrm{TS}=-18)$ than the LDDE.
}

As made clear from this work, the evolution of the BL Lac class is complex. We found that the evolution of BL Lac objects selected by Fermi can be described with a LDDE model similar to the one used for FSRQs (Ajello et al. 2012). Indeed, luminous BL Lac objects $\left(\sim 10^{47} \mathrm{erg} \mathrm{s}^{-1}\right)$ evolve as strongly $\left(p_{1} \sim 7\right)$ as FSRQs (see Section 4.2). However, the evolution of BL Lac objects slows down with luminosity, becoming negative for objects with $L_{\gamma} \leqslant 10^{45.5} \mathrm{erg} \mathrm{s}^{-1}$.

Subdividing the sample in HSP, ISP, and LSP objects, we find that the negative evolution is in fact isolated to the HSP population, while the ISP and LSP evolve positively from the lowest luminosities. Our analysis thus confirms results based on samples dominated by HSP objects (e.g., Giommi et al. 1999; Beckmann et al. 2003). We tested if different slopes of the LF (Equation (12)) respectively for HSPs and ISPs+LSPs could be compatible with a common (e.g., positive) shape of the evolution. We find that, while it seems HSPs have a slightly flatter LF (at redshift $\approx 0$ ) than ISPs+LSPs, imposing a common shape of the evolution to the whole population substantially worsen the fit (by $\sim 10 \sigma$ ). On the other hand, allowing HSPs and ISPs+LSPs to have different evolutions reproduces the negativepositive dichotomy. ${ }^{19}$ We can also exclude that the negative evolution scenario is caused by inadequate redshift coverage (incompleteness) or by the fact that HSPs are not detected to sufficiently large redshifts (sensitivity limit). Indeed, from our set of Monte Carlo simulations we find that $\sim 30 \%$ and $\sim 7 \%$ of all the HSPs detected by Fermi lie respectively at $z>1$ and $z>1.5$. Moreover, the effect of the extragalactic background light (EBL, see Section 6.2) is not severe and does not bias either the measured fluxes or the photon indices in the $0.1-100 \mathrm{GeV}$ band. In order to exclude the notion that the negative evolution of low-luminosity BL Lac objects (and HSPs) is caused by the incompleteness of the sample used here (see Section 2), we explore a worst case scenario assuming that all $\sim 20$ unassociated sources are BL Lac objects lying in the $0.2-0.7$ redshift range. A large population of BL Lac objects at intermediate redshifts $(z \sim 0.5$, see left panel of Figure 11) would be needed to invert the negative evolution. Using actual fluxes and photon indices drawn from the 23 unclassified possible AGNs, assuming that all are HSP and drawing random redshifts in the critical $0.2-0.7$ range, we find that only a relatively small fraction $(\sim 12 \%)$ could be HSPs with $\log L_{\gamma}<45.5$. Accordingly, even in this worstcase scenario we find that these missing identifications cannot significantly alter our measurement of negative evolution for this subclass.

The slowing down of the evolution with decreasing source luminosity has been observed in many kinds of AGNs, including the population of radio galaxies (Longair 1966; Schmidt 1972; Willott et al. 2001), but an inversion of the evolution at very low luminosity as observed here is difficult to interpret. While the close connection between the FSRQ and LSP classes is quite apparent, it is less obvious that this trend can be extended to the

\footnotetext{
19 In this test we defined the luminosity function as the sum of two different functions representing the HSP and ISP+LSP populations. For the HSP population we adopted a single power law in luminosity and a PLE model with $e(z)=(1+z)^{k}$ while we adopted the PLE model with $\beta=0$ described in Section 3.2. For the HSP and ISP+LSP populations we found a slope (in luminosity) of the luminosity function of $2.04 \pm 0.08$ and $2.35 \pm 0.10$, respectively, while for the evolutionary factor we found $k=-0.9 \pm 0.3$ (for HSPs), $k=12.4 \pm 0.7$, and $\gamma=-0.19 \pm 0.01$ (for the ISPs+LSPs). These results imply that the two populations have a similar slope in luminosity, but a different form of the evolution that is confirmed to be negative for HSPs and positive (with a redshift peak at $\approx 1.3$ ) for ISPs+LSPs.
} 
HSP BL Lac objects. However, one may interpret this spectral sequence as a progression caused by the gradual depletion of an AGN's gas reservoir via accretion (e.g., Cavaliere \& D'Elia 2002; Böttcher \& Dermer 2002). In this context an LSP object would transition from disk-powered jet production (at high accretion rates) through the ISP class to a HSP BL Lac object with low accretion rates and a radiatively inefficient accretion flow. In LSPs, strong cooling due to the circumnuclear radiation fields would limit the maximum energy reached by the accelerated electrons. For the HSPs, due to the decreased cooling efficiency, particles would be accelerated to much larger energies, which would translate into a peak frequency of the synchrotron component that moves from $10^{13} \mathrm{~Hz}$ up to $10^{17} \mathrm{~Hz}$. This reproduces the paradigm of the blazar sequence (Ghisellini et al. 1998; Fossati et al. 1998).

The activity of FSRQs, if triggered by galaxy merging events as is common for high-luminosity quasars, would be short lived ( $\tau \sim 0.1 \mathrm{Gyr}$ ) and followed by the low-accretion regime of HSP-type BL Lac objects, which can be sustained for much longer times $(\tau \sim 5--7$ Gyr Cavaliere \& D'Elia 2002). In the high-redshift universe, where gas was abundant, galaxy merging favors the activity of FSRQs. As the universe expands, galaxy merging becomes infrequent and most of the FSRQs/LSPs finish consuming their fuel reserve, transitioning to a long-lasting low accretion regime. If the HSPs are indeed starved LSP objects, then one should observe an increase in the space density of BL Lac objects with only a slight lag (since $\tau \sim 0.1$ Gyr for FSRQs) from the decrease in the space density of FSRQs. Figure 10 can been seen as supporting this picture. Indeed at $z \geqslant 1.5$ the number density of HSPs decreases in a similar way to that of FSRQs and LSPs and ISPs objects. At $z<0.5$ when the FSRQs turn off, the space density of HSPs, and in particular the low luminosity HSP, quickly increases.

This scenario is attractive but still speculative. At present we lack a quantitative comparison between the space densities of the FSRQ+LSP objects and the (possibly remnant) population of HSP. Certainly different beaming characteristics (and their potential evolution with redshift) can affect the estimated populations and complicate this comparison. There may also be differences between the low- and high-peaked sources in the typical black hole mass or host galaxy environment. Nevertheless, the correlation of opposing evolutionary trends found here points to a possible connection between these AGN populations.

\subsection{Softening of Blazar Spectra with Redshift}

In Sections 4.1 and 4.2 we found that Fermi blazar spectra soften with increasing luminosity. In particular, all the best-fit models have BL Lac spectra softening at high luminosity. The average photon index changes from $\sim 2.0$ to $\sim 2.2$ when the luminosity changes from $10^{44} \mathrm{erg} \mathrm{s}^{-1}$ to $10^{48} \mathrm{erg} \mathrm{s}^{-1}$.

The left panel of Figure 12 shows the deconvolved intrinsic photon index distributions for three different luminosity classes. The deconvolution was performed with the method outlined in Section 3.1 (see Equation (9)). The $y$-axis reports the integral over redshift and luminosity of Equation (1) (essentially $d N / d \Gamma)$. The trend of the average softening of the BL Lac spectra with increasing luminosity is apparent even though the dynamic range is small: i.e., the index changes by only $\Delta \Gamma \approx 0.2$ in 4 orders of magnitudes in luminosity. The right panel of Figure 12 shows the photon index-luminosity plane as predicted by the best-fit $\mathrm{LF}^{20}$ The correlation of the photon index with luminosity is very clear. Both of these figures include corrections for all known selection effects, so we infer that this trend is directly apparent in the sources (although it is strongly amplified in the observed sample through selection effects).

If selection effects are not properly taken into account, a spurious index-luminosity correlation can be artificially introduced because of the energy dependence of the Fermi-LAT pointspread function (Atwood et al. 2009) which favors the detection at low fluxes of sources with a hard spectrum (see Figure 1 in Abdo et al. 2010c). However, the analysis of the source count distribution as a function of the photon index did not reveal any significant correlation between flux and photon index (Abdo et al. 2010c; Singal et al. 2012).

Finally, a spurious luminosity-index correlation might be produced by the absorption of high-energy photons by the EBL. The EBL attenuation would make measured spectra steeper than intrinsic, preferentially affecting high-redshift (and thus highluminosity) sources. We checked to see if this produced the observed trend by simulating $\sim 1000$ spectra in the $0.1-100 \mathrm{GeV}$ band using a power-law model with a photon index of 2.0. Fluxes and redshifts were drawn from the observed sample of BL Lac objects, and EBL absorption was applied using models (Franceschini et al. 2008; Finke et al. 2010; Domínguez et al. 2011) in agreement with Fermi observations of the EBL attenuation (Ackermann et al. 2012b). The result of this analysis, reported in Figure 13, shows that the EBL effect on measured photon indices (in the $0.1-100 \mathrm{GeV}$ band) is minor. While the photon index of BL Lac objects with $\log L_{\gamma}>47.5 \mathrm{erg} \mathrm{s}^{-1}$ is modified by the EBL attenuation by $\Delta \Gamma \sim 0.055$, the index of all sources below that luminosity is basically unaffected. Thus, we conclude that the observed index-luminosity correlation is not an artifact of selection effects or cosmic EBL absorption, but intrinsic to the sources.

Ghisellini et al. (2009) was the first to note (although without accounting for selection effects) that a correlation between index and $\gamma$-ray luminosity seemed to exist for BL Lac objects and FSRQs detected by Fermi. They proposed that the $0.1-100 \mathrm{GeV}$ luminosity of $10^{47} \mathrm{erg} \mathrm{s}^{-1}$ that separates hard BL Lac objects from soft FSRQs could be associated with a transition in the accretion flow from radiatively inefficient (e.g., Narayan et al. 1997) to optically thick radiatively efficient (Shakura \& Sunyaev 1973).

The picture seems to be slightly more complex. FSRQs stand as a monolithic population for which there is no correlation between photon index and luminosity (Ajello et al. 2012). This is likely due to the fact that at $\mathrm{GeV}$ energies their spectrum is dominated by the external Compton emission (Dermer \& Schlickeiser 1993). On the other hand, the index-luminosity correlation for BL Lac objects as argued above has a significant intrinsic component. This points toward the fact that particles in luminous BL Lac objects cool more efficiently than in low-luminosity objects, which is in agreement with the results of Finke (2013) who finds an anticorrelation between the Compton dominance and the frequency at the peak of the synchrotron component.

The correlation between index and luminosity reported by Ghisellini et al. (2009) is much stronger than that which we find here $\left(\Delta \Gamma / \Delta \log L_{\gamma} \sim 0.25\right.$ versus $\left.\sim 0.06\right)$; this was thus likely dominated by the uncorrected selection effects. It does

\footnotetext{
20 Each point of the index-luminosity plane reports the number of BL Lac objects that would be visible in the whole sky from an ideal telescope that suffered no selection effects.
} 

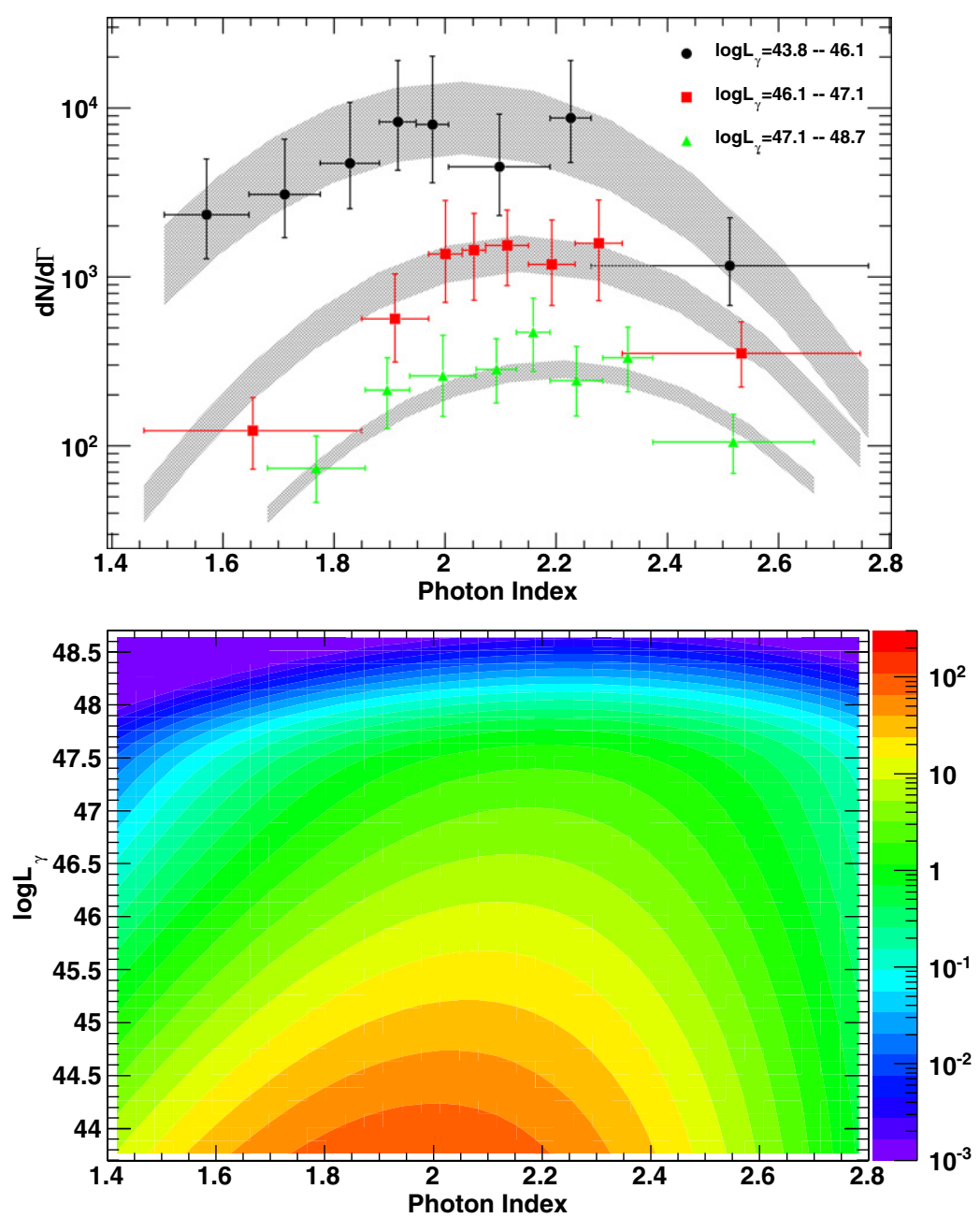

Figure 12. Top panel: deconvolved index distribution for three luminosity classes of Fermi BL Lac objects. Note the shift in the average of the distribution with luminosity. Bottom panel: deconvolved photon index-luminosity plane for the Fermi BL Lac objects (in units of number of BL Lac objects per bin of luminosity and index).

(A color version of this figure is available in the online journal.)

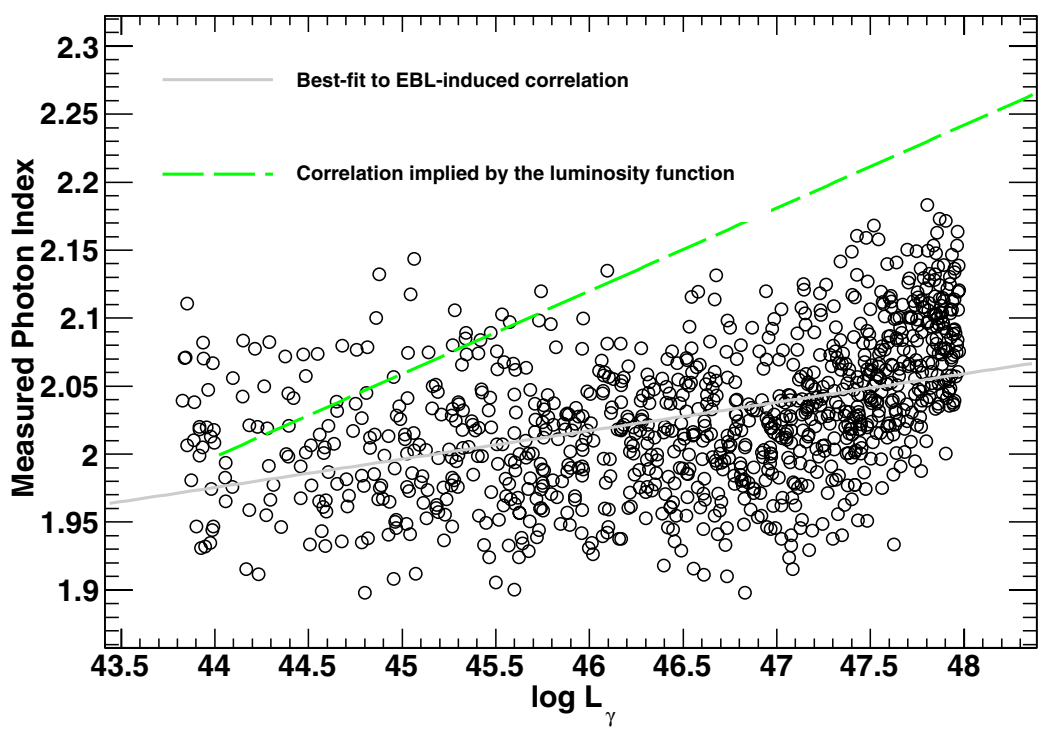

Figure 13. Measured photon index vs. measured luminosity for a sample of 1000 power-law spectra (with $\Gamma=2)$ with fluxes and redshifts randomly drawn from the sample of BL Lac objects used here. An EBL attenuation using the model of Franceschini et al. (2008) was applied to all the spectra. The measured quantities (index, flux, and luminosity) were derived by fitting source spectra with a power law. The solid line shows the best linear fit to the observed data points. The dashed line shows the correlation between photon index and luminosity found by the best-fit LDDE model in Section 4.2.

(A color version of this figure is available in the online journal.) 
not seem to be the case that luminous and hard BL Lac objects exist in such numbers to destroy the correlation as suggested by Giommi et al. (2013), although a few such objects are indeed seen in our sample. Hard luminous BL Lac objects exist (see Figure 12) as predicted by the Fermi best-fit LF, but they are rare, representing the tail of the $d N / d L_{\gamma} d \Gamma$ distribution.

\subsection{The Contribution to the Isotropic Gamma-Ray Background}

This analysis has important consequences for the understanding of the IGRB Fichtel et al. (1975); Sreekumar et al. (1998); Abdo et al. (2010b) whose origin is still unclear (Abdo et al. 2010c; Ajello et al. 2012).

A simple integration of the LF yields the diffuse emission arising from the unresolved BL Lac class (in the $0.1-100 \mathrm{GeV}$ band) as $8.0_{-1.3}^{+2.0} \times 10^{-7} \mathrm{ph} \mathrm{cm}^{-2} \mathrm{~s}^{-1} \mathrm{sr}^{-1}$, which represents $7.7_{-1.3}^{+2.0} \%$ of the intensity measured by Fermi. The slightly disfavored PLE model predicts that BL Lac objects produce $1.07_{-0.17}^{+0.21} \times 10^{-6} \mathrm{ph} \mathrm{cm}^{-2} \mathrm{~s}^{-1} \mathrm{sr}^{-1}$ and thus $10.3 \% \pm 2 \%$ of the IGRB. It thus seems clear that BL Lac objects do not account for more than $\sim 10 \%-15 \%$ of the IGRB.

While this might seem to represent a small number, the large density of hard sources present in the nearby universe, as predicted by the LF makes the spectrum of the diffuse emission arising from the BL Lac class harder than that of the IGRB. Since this depends on the assumed spectral models for different BL Lac classes and on the EBL model, the actual contribution from the common extreme HSP sources may be larger. The exact energy-dependent derivation is left to a future publication.

\section{SUMMARY}

This work relies on a complete sample of 211 BL Lac objects, detected by Fermi during its first year of operations, to deepen our knowledge of this elusive, yet very important, blazar population. Our findings can be summarized as follows.

1. The typical redshift completeness of any BL Lac sample is $<50 \%$. The Fermi sample is no exception with only 103 BL Lac objects (out of 211) having a spectroscopic redshift measurement. ${ }^{21}$ Using four different techniques (described in Section 3.3) we were able to provide quantitative constraints on the redshift of an additional 104 objects, making this the largest and most complete sample of BL Lac objects available in the literature. We find that most of the objects without a spectroscopic redshift (and thus $\sim$ half of the BL Lac population) lie at $z>0.5-0.7$, which is larger than the typical spectroscopic limit reached for BL Lac objects.

2. Independently of the functional form used to represent the data, we find that the BL Lac population displays (as found for other classes of AGNs) a speed of evolution that depends on luminosity, with high-luminosity sources evolving faster than low-luminosity sources. The negative evolution (i.e., more BL Lac objects at lower than higher redshifts) of the low-luminosity BL Lac objects is a major result of this work. We find that HSPs are certainly responsible for most, if not all, of the detected negative evolution. This confirms previous claims of negative evolution based on samples of X-ray-selected BL Lac objects, which contained a large fraction of HSPs (Rector et al. 2000; Beckmann et al. 2003).

\footnotetext{
21 A similar fraction also holds for the 2LAC sample of 423 BL Lac objects
} (Ackermann et al. 2011).
3. This work allows us to explore the link between the BL Lac and the FSRQ families of blazars. The local $(z \approx 0)$ LF of BL Lac overlaps and connects smoothly to that of FSRQs, highlighting the similarity between the two classes with BL Lac objects having on average lower luminosity (and thus very likely lower Lorentz factors) than FSRQs. This last aspect is confirmed by the analysis of the intrinsic LF that allows us, using a simple beaming model, to derive the distributions of Lorentz factors and viewing angles. FSRQs and BL Lac objects have a similar distribution of Lorentz factors (i.e., a power-law distribution with an index $\approx-2.5$ ), but the distribution of BL Lac objects extends to slower jet speeds, implying that the jets of BL Lac objects are, on average, seen under larger angles than those of FSRQs $\left(\sim 5^{\circ}\right.$ for BL Lac objects versus $\sim 2{ }^{\circ}$ for FSRQs).

4. One of the most interesting findings of this work is the evidence supporting the genetic link between FSRQs and BL Lac objects as proposed by Cavaliere \& D'Elia (2002) and Böttcher \& Dermer (2002). In this scenario BL Lac objects represent the final (gas-starved, inefficiently accreting) and long-lasting phase of an earlier, short-lived, merger-driven, gas-rich epoch (the FSRQ). The sudden increase in the space density of BL Lac objects (driven in particular by the HSPs) at the same epoch as the turn off of FSRQs corroborates the idea of a transition from the FSRQ to the BL Lac class. To investigate further the details of this transition would require, for both classes, a robust beaming correction and knowledge of the black hole mass and host galaxy environment, which are at present not well constrained.

5. The study of the LF shows that the spectra of BL Lac objects at $\mathrm{GeV}$ energies soften with increasing luminosity even after correcting for the substantial selection effects. The effect is not as dramatic as reported in the literature (e.g., Ghisellini et al. 2009), but might still be caused by the fact that particles in luminous BL Lac objects cool more efficiently than in low-luminosity objects.

6. Unresolved BL Lac objects contribute $\sim 10 \%-15 \%$ of the IGRB measured by Fermi (Abdo et al. 2010b). However, the large density of hard sources at low redshift, as implied from the LF derived in this work, will certainly increase the contribution of BL Lac objects to the IGRB at $>10 \mathrm{GeV}$. A confirmation of this is already available in the study of the $>10 \mathrm{GeV}$ sources detected by Fermi (Ackermann et al. 2013).

The comments from an expert referee are gratefully acknowledged. M.A. acknowledges generous support from NASA grant NNH09ZDA001N for the study of the origin of the extragalactic gamma-ray background, and hospitality at Goddard and NRL while writing part of this work. R.W.R acknowledges NASA grants NNX08AW30G and NNX11A044G and extensive consultation with the OVRO Fermi group. D.G. acknowledges financial contribution from the agreement ASI-INAF $\mathrm{I} / 009 / 10 / 0$. M.A. is indebted to J. Wall for originally sharing the details of the ML method used in this and other works.

The Fermi-LAT Collaboration acknowledges generous ongoing support from a number of agencies and institutes that have supported both the development and the operation of the LAT as well as scientific data analysis. These include the National Aeronautics and Space Administration and the Department of Energy in the United States; the Commissariat à l'Energie Atomique and the Centre National de la Recherche Scientifique/Institut 
National de Physique Nucléaire et de Physique des Particules in France; the Agenzia Spaziale Italiana and the Istituto Nazionale di Fisica Nucleare in Italy; the Ministry of Education, Culture, Sports, Science and Technology (MEXT), High Energy Accelerator Research Organization (KEK), and Japan Aerospace Exploration Agency (JAXA) in Japan; and the K. A. Wallenberg
Foundation, the Swedish Research Council, and the Swedish National Space Board in Sweden. Additional support for science analysis during the operations phase is gratefully acknowledged from the Istituto Nazionale di Astrofisica in Italy and the Centre National d'Études Spatiales in France.

Facility: Fermi (LAT)

\section{APPENDIX}

\section{A.1. Table with Redshift Constraints}

Table 5 reports the 211 BL Lac objects used in this work with all the available redshift constraints (with the exception of the exclusion functions). The sample and the nature of the redshift constraints are described in Sections 2 and 3.3.

Table 5

The 211 BL Lac Objects Detected by Fermi Used for This Analysis

\begin{tabular}{|c|c|c|c|c|c|c|c|c|}
\hline NAME & $\operatorname{Flux}_{100^{a}}$ & Photon Index & $z^{\mathrm{b}}$ & Photo- $z^{c}$ & $z_{L} L^{\mathrm{d}}$ & $z_{\text {MAX }}{ }^{\mathrm{e}}$ & Photo- $z_{U} L^{\mathrm{f}}$ & SED CLASS $^{\mathrm{g}}$ \\
\hline 1FGL J0006.9+4652 & $4.35 \pm 0.79$ & $2.50 \pm 0.12$ & $\cdots$ & $\cdots$ & $\cdots$ & $\cdots$ & $\cdots$ & ISP \\
\hline 1FGL J0021.7-2556 & $1.22 \pm 0.41$ & $2.13 \pm 0.16$ & $\cdots$ & $\cdots$ & 0.56 & 1.63 & 1.44 & ISP \\
\hline 1FGL J0022.2-1850 & $0.45 \pm 0.16$ & $1.64 \pm 0.13$ & $\cdots$ & $\cdots$ & 0.77 & 1.64 & 1.38 & HSP \\
\hline 1FGL J0033.5-1921 & $2.03 \pm 0.32$ & $1.89 \pm 0.07$ & $\cdots$ & $\cdots$ & $0.50^{\mathrm{h}}$ & 1.77 & $\cdots$ & HSP \\
\hline 1FGL J0035.1+1516 & $0.70 \pm 0.23$ & $1.72 \pm 0.12$ & $\cdots$ & 1.28 & $\cdots$ & 1.65 & $\cdots$ & HSP \\
\hline 1FGL J0038.0+1236 & $1.41 \pm 0.59$ & $2.23 \pm 0.19$ & 0.089 & $\cdots$ & $\cdots$ & 1.76 & $\cdots$ & HSP \\
\hline 1FGL J0045.3+2127 & $1.39 \pm 0.38$ & $1.86 \pm 0.11$ & $\cdots$ & $\cdots$ & $\cdots$ & 1.78 & 1.06 & HSP \\
\hline 1FGL J0050.6-0928 & $7.39 \pm 0.64$ & $2.20 \pm 0.05$ & 0.635 & $\cdots$ & $\cdots$ & 2.18 & $\cdots$ & ISP \\
\hline 1FGL J0100.2+0747 & $1.90 \pm 0.36$ & $1.90 \pm 0.09$ & $\cdots$ & $\cdots$ & $\cdots$ & 4.01 & $\cdots$ & ISP/HSP \\
\hline 1FGL J0105.7+3930 & $4.80 \pm 0.90$ & $2.70 \pm 0.14$ & 0.440 & $\cdots$ & $\cdots$ & 2.68 & $\cdots$ & $\cdots$ \\
\hline 1FGL J0109.0+1816 & $0.76 \pm 0.35$ & $2.00 \pm 0.19$ & 0.443 & $\cdots$ & $\cdots$ & 2.48 & $\cdots$ & HSP \\
\hline 1FGL J0114.4+1327 & $3.81 \pm 0.76$ & $2.66 \pm 0.14$ & $\cdots$ & $\cdots$ & $\cdots$ & 1.63 & 1.22 & ISP/HSP \\
\hline 1FGL J0115.5+2519 & $1.49 \pm 0.42$ & $2.02 \pm 0.12$ & $\cdots$ & $\cdots$ & 0.27 & 1.63 & 1.45 & HSP \\
\hline 1FGL J0115.7+0357 & $1.19 \pm 0.40$ & $2.03 \pm 0.15$ & 0.913 & $\cdots$ & 0.14 & 1.62 & 1.25 & $\cdots$ \\
\hline 1FGL J0120.5-2700 & $3.90 \pm 0.45$ & $2.03 \pm 0.06$ & $\cdots$ & $\cdots$ & 0.56 & 1.76 & $\ldots$ & ISP \\
\hline 1FGL J0136.5+3905 & $2.86 \pm 0.39$ & $1.80 \pm 0.06$ & $\cdots$ & $\cdots$ & $\cdots$ & 1.65 & $\cdots$ & HSP \\
\hline 1FGL J0141.7-0929 & $1.85 \pm 0.45$ & $2.16 \pm 0.12$ & 0.735 & $\cdots$ & 0.50 & 2.17 & $\cdots$ & ISP \\
\hline 1FGL J0144.6+2703 & $4.77 \pm 0.65$ & $2.22 \pm 0.08$ & $\cdots$ & $\cdots$ & 0.71 & 1.66 & $\cdots$ & ISP \\
\hline 1FGL J0154.1+0823 & $1.68 \pm 0.39$ & $1.97 \pm 0.10$ & 0.681 & $\cdots$ & 0.34 & 1.64 & 1.37 & ISP \\
\hline 1FGL J0155.0+4433 & $0.95 \pm 0.55$ & $2.10 \pm 0.23$ & $\cdots$ & $\cdots$ & 0.39 & 1.63 & $\cdots$ & ISP/HSP \\
\hline 1FGL J0158.0-3931 & $2.19 \pm 0.52$ & $2.34 \pm 0.14$ & $\cdots$ & $\cdots$ & $\cdots$ & 2.15 & 1.35 & ISP \\
\hline 1FGL J0159.5+1047 & $1.28 \pm 0.19$ & $1.95 \pm 0.06$ & 0.195 & $\cdots$ & $\cdots$ & 1.76 & $\cdots$ & HSP \\
\hline 1FGL J0159.7-2741 & $0.98 \pm 0.30$ & $2.06 \pm 0.14$ & $\cdots$ & $\cdots$ & 0.58 & 1.78 & 1.05 & ISP \\
\hline 1FGL J0203.5+3044 & $4.91 \pm 0.85$ & $2.74 \pm 0.13$ & 0.761 & $\cdots$ & $\cdots$ & 2.72 & $\cdots$ & $\cdots$ \\
\hline 1FGL J0209.3-5229 & $1.80 \pm 0.48$ & $1.94 \pm 0.11$ & $\cdots$ & $\cdots$ & $\cdots$ & 2.18 & 1.18 & HSP \\
\hline 1FGL J0210.6-5101 & $14.63 \pm 0.95$ & $2.37 \pm 0.04$ & 0.999 & $\cdots$ & $\cdots$ & $\cdots$ & $\cdots$ & ISP \\
\hline 1FGL J0211.2+1049 & $3.86 \pm 0.69$ & $2.27 \pm 0.09$ & $\cdots$ & $\cdots$ & $\cdots$ & 1.67 & $\cdots$ & ISP \\
\hline 1FGL J0213.2+2244 & $1.29 \pm 0.40$ & $1.95 \pm 0.13$ & 0.459 & $\cdots$ & $\cdots$ & 2.66 & $\cdots$ & HSP \\
\hline 1FGL J0217.9-6630 & $1.19 \pm 0.46$ & $2.07 \pm 0.17$ & $\cdots$ & $\cdots$ & 0.67 & 1.88 & 1.25 & HSP \\
\hline 1FGL J0222.6+4302 & $21.51 \pm 1.03$ & $1.94 \pm 0.02$ & $\cdots$ & $\cdots$ & $\cdots$ & 1.67 & $\cdots$ & ISP \\
\hline 1FGL J0238.6+1637 & $43.54 \pm 1.10$ & $2.15 \pm 0.02$ & 0.940 & $\cdots$ & $\cdots$ & $\cdots$ & $\cdots$ & ISP \\
\hline 1FGL J0238.6-3117 & $0.96 \pm 0.35$ & $2.07 \pm 0.17$ & 0.232 & $\cdots$ & $\cdots$ & 1.63 & 1.02 & HSP \\
\hline 1FGL J0250.4+1715 & $1.28 \pm 0.40$ & $2.13 \pm 0.13$ & 0.612 & $\cdots$ & $\cdots$ & $\cdots$ & 3.10 & $\cdots$ \\
\hline 1FGL J0303.5-2406 & $4.71 \pm 0.41$ & $2.00 \pm 0.05$ & 0.260 & $\cdots$ & $\cdots$ & $\cdots$ & $\cdots$ & HSP \\
\hline 1FGL J0315.9-2609 & $0.32 \pm 0.16$ & $1.62 \pm 0.17$ & 0.443 & $\cdots$ & $\cdots$ & $\cdots$ & $\cdots$ & HSP \\
\hline 1FGL J0316.1+0904 & $1.73 \pm 0.42$ & $1.78 \pm 0.09$ & $\cdots$ & $\cdots$ & $\cdots$ & 1.66 & $\cdots$ & HSP \\
\hline 1FGL J0319.7+1847 & $0.51 \pm 0.23$ & $1.65 \pm 0.16$ & 0.190 & $\cdots$ & $\cdots$ & $\cdots$ & $\cdots$ & HSP \\
\hline 1FGL J0322.1+2336 & $4.26 \pm 0.92$ & $2.41 \pm 0.12$ & $\cdots$ & $\cdots$ & $\cdots$ & $\cdots$ & $\cdots$ & HSP \\
\hline 1FGL J0323.7-0106 & $0.34 \pm 0.16$ & $1.59 \pm 0.17$ & 0.392 & $\cdots$ & $\cdots$ & 2.17 & 1.54 & HSP \\
\hline 1FGL J0326.2+0222 & $1.94 \pm 0.53$ & $2.21 \pm 0.13$ & 0.147 & $\cdots$ & $\cdots$ & $\cdots$ & $\cdots$ & HSP \\
\hline 1FGL J0334.2-4010 & $7.85 \pm 0.10$ & $2.34 \pm 0.01$ & 1.357 & $\cdots$ & 1.21 & 2.05 & $\cdots$ & ISP \\
\hline 1FGL J0334.4-3727 & $2.59 \pm 0.18$ & $2.09 \pm 0.04$ & $\cdots$ & $\cdots$ & $\cdots$ & 1.92 & 1.34 & ISP \\
\hline 1FGL J0354.6+8009 & $7.76 \pm 0.90$ & $2.58 \pm 0.08$ & $\cdots$ & $\cdots$ & $\cdots$ & $\cdots$ & $\cdots$ & ISP \\
\hline 1FGL J0416.8+0107 & $0.80 \pm 0.50$ & $1.96 \pm 0.24$ & 0.287 & $\cdots$ & $\cdots$ & $\cdots$ & $\cdots$ & HSP \\
\hline 1FGL J0428.6-3756 & $31.07 \pm 0.89$ & $2.13 \pm 0.02$ & 1.111 & $\cdots$ & $\cdots$ & $\cdots$ & $\cdots$ & ISP \\
\hline 1FGL J0434.1-2018 & $1.67 \pm 0.46$ & $2.31 \pm 0.15$ & 0.928 & $\cdots$ & $\cdots$ & 2.43 & $\cdots$ & ISP \\
\hline 1FGL J0448.5-1633 & $1.05 \pm 0.37$ & $1.97 \pm 0.15$ & $\cdots$ & $\cdots$ & $\cdots$ & 1.63 & 1.25 & HSP \\
\hline 1FGL J0449.5-4350 & $10.40 \pm 0.55$ & $1.99 \pm 0.03$ & 0.205 & $\cdots$ & $\cdots$ & $\cdots$ & $\cdots$ & HSP \\
\hline 1FGL J0507.9+6738 & $1.37 \pm 0.20$ & $1.73 \pm 0.06$ & 0.340 & $\cdots$ & $\cdots$ & 2.51 & $\cdots$ & HSP \\
\hline 1FGL J0509.3+0540 & $8.18 \pm 0.87$ & $2.31 \pm 0.06$ & 0.336 & $\cdots$ & $\cdots$ & 1.66 & 1.24 & ISP \\
\hline
\end{tabular}


Table 5

(Continued)

\begin{tabular}{|c|c|c|c|c|c|c|c|c|}
\hline NAME & Flux $_{100}{ }^{a}$ & Photon Index & $z^{\mathrm{b}}$ & Photo- $z^{\mathrm{c}}$ & $z_{L} L^{\mathrm{d}}$ & $z_{\text {MAX }^{\mathrm{e}}}$ & Photo- $z_{U} L^{\mathrm{f}}$ & SED CLASS \\
\hline 1FGL J0516.7-6207 & $5.56 \pm 0.01$ & $2.28 \pm 0.00$ & 1.300 & $\cdots$ & $\cdots$ & 1.87 & $\cdots$ & ISP \\
\hline 1FGL J0536.2-3348 & $5.54 \pm 0.70$ & $2.37 \pm 0.08$ & $\cdots$ & $\cdots$ & $\ldots$ & 2.17 & 1.16 & HSP \\
\hline 1FGL J0538.8-4404 & $38.22 \pm 1.08$ & $2.28 \pm 0.02$ & 0.892 & $\cdots$ & $\cdots$ & $\cdots$ & $\cdots$ & ISP \\
\hline 1FGL J0543.8-5531 & $0.97 \pm 0.31$ & $1.75 \pm 0.12$ & $0.271^{\mathrm{h}}$ & $\cdots$ & $\cdots$ & 2.57 & 1.08 & HSP \\
\hline 1FGL J0616.9+5701 & $1.63 \pm 0.48$ & $2.06 \pm 0.13$ & $\cdots$ & $\cdots$ & 0.80 & 3.94 & $\cdots$ & ISP \\
\hline 1FGL J0617.7-1718 & $1.14 \pm 0.45$ & $1.98 \pm 0.15$ & 0.098 & $\cdots$ & $\cdots$ & 1.75 & $\cdots$ & ISP \\
\hline 1FGL J0700.4-6611 & $5.61 \pm 0.01$ & $2.13 \pm 0.00$ & $\cdots$ & $\cdots$ & $\cdots$ & 1.92 & 1.46 & ISP \\
\hline 1FGL J0706.5+3744 & $1.82 \pm 0.55$ & $2.19 \pm 0.14$ & $\ldots$ & $\cdots$ & $\cdots$ & 1.63 & $\ldots$ & HSP \\
\hline 1FGL J0707.3+7742 & $2.48 \pm 0.29$ & $2.28 \pm 0.06$ & $\cdots$ & $\cdots$ & $\cdots$ & 1.76 & $\cdots$ & ISP \\
\hline 1FGL J0710.6+5911 & $0.24 \pm 0.12$ & $1.50 \pm 0.18$ & 0.125 & $\cdots$ & $\cdots$ & $\cdots$ & $\cdots$ & HSP \\
\hline 1FGL J0711.4+4731 & $2.91 \pm 0.68$ & $2.52 \pm 0.14$ & 1.292 & $\cdots$ & $\cdots$ & $\cdots$ & $\cdots$ & ISP \\
\hline 1FGL J0712.7+5033 & $2.86 \pm 0.47$ & $2.07 \pm 0.08$ & 0.502 & $\cdots$ & $\cdots$ & 1.67 & $\cdots$ & ISP \\
\hline 1FGL J0721.9+7120 & $17.39 \pm 0.80$ & $2.15 \pm 0.03$ & $\ldots$ & $\cdots$ & $\cdots$ & 2.61 & $\cdots$ & ISP \\
\hline 1FGL J0738.2+1741 & $5.08 \pm 0.52$ & $2.06 \pm 0.05$ & $\ldots$ & $\cdots$ & 0.42 & 1.80 & $1.30^{\mathrm{i}}$ & HSP \\
\hline 1FGL J0752.8+5353 & $0.88 \pm 0.34$ & $1.95 \pm 0.16$ & 0.730 & $\cdots$ & $\cdots$ & 1.94 & $\cdots$ & ISP \\
\hline 1FGL J0757.2+0956 & $5.30 \pm 0.69$ & $2.44 \pm 0.08$ & 0.266 & $\cdots$ & $\cdots$ & $\cdots$ & $\cdots$ & ISP \\
\hline 1FGL J0804.7+7534 & $0.71 \pm 0.30$ & $1.79 \pm 0.15$ & 0.121 & $\cdots$ & $\cdots$ & $\cdots$ & $\cdots$ & HSP \\
\hline 1FGL J0809.5+5219 & $2.07 \pm 0.48$ & $1.99 \pm 0.11$ & 0.137 & $\cdots$ & $\cdots$ & 2.22 & $\cdots$ & HSP \\
\hline 1FGL J0811.1-7527 & $1.39 \pm 0.39$ & $1.86 \pm 0.11$ & $\ldots$ & $\cdots$ & 0.69 & 1.91 & 1.40 & ISP \\
\hline 1FGL J0811.2+0148 & $3.58 \pm 0.70$ & $2.56 \pm 0.13$ & 1.148 & $\cdots$ & $\ldots$ & $\ldots$ & $\cdots$ & ISP \\
\hline 1FGL J0815.0+6434 & $3.10 \pm 0.63$ & $2.31 \pm 0.11$ & 0.239 & $\cdots$ & $\cdots$ & 1.64 & $\cdots$ & ISP \\
\hline 1FGL J0818.2+4222 & $12.19 \pm 0.71$ & $2.17 \pm 0.04$ & $\cdots$ & $\cdots$ & $\cdots$ & 2.47 & $\cdots$ & ISP \\
\hline 1FGL J0825.9+0309 & $0.51 \pm 0.28$ & $1.88 \pm 0.21$ & 0.505 & $\cdots$ & $\cdots$ & 3.21 & $\cdots$ & ISP \\
\hline 1FGL J0831.6+0429 & $7.18 \pm 0.76$ & $2.49 \pm 0.07$ & 0.174 & $\cdots$ & $\cdots$ & 2.19 & $\cdots$ & ISP \\
\hline 1FGL J0844.0+5314 & $0.51 \pm 0.23$ & $1.90 \pm 0.18$ & $\ldots$ & $\cdots$ & $\cdots$ & 2.51 & $\cdots$ & ISP \\
\hline 1FGL J0847.2+1134 & $0.23 \pm 0.10$ & $1.49 \pm 0.16$ & 0.198 & $\ldots$ & $\cdots$ & 2.17 & $\cdots$ & HSP \\
\hline 1FGL J0854.8+2006 & $5.37 \pm 0.55$ & $2.20 \pm 0.06$ & 0.306 & $\cdots$ & $\cdots$ & $\cdots$ & $\cdots$ & ISP \\
\hline 1FGL J0856.6-1105 & $5.70 \pm 0.71$ & $2.34 \pm 0.07$ & $\ldots$ & $\ldots$ & 1.40 & 2.18 & 1.54 & ISP \\
\hline 1FGL J0902.4+2050 & $1.65 \pm 0.44$ & $2.11 \pm 0.13$ & $\cdots$ & $\cdots$ & $\cdots$ & 2.18 & 1.21 & ISP \\
\hline 1FGL J0905.5+1356 & $0.90 \pm 0.35$ & $1.94 \pm 0.16$ & $\cdots$ & $\cdots$ & $\cdots$ & 1.64 & 1.35 & HSP \\
\hline 1FGL J0910.7+3332 & $1.69 \pm 0.48$ & $2.26 \pm 0.14$ & 0.354 & $\cdots$ & $\cdots$ & 1.77 & $\cdots$ & HSP \\
\hline 1FGL J0915.7+2931 & $1.67 \pm 0.11$ & $1.95 \pm 0.03$ & $\ldots$ & $\cdots$ & $\cdots$ & 1.69 & $\cdots$ & HSP \\
\hline 1FGL J0945.6+5754 & $1.50 \pm 0.46$ & $2.21 \pm 0.15$ & 0.229 & $\cdots$ & $\cdots$ & 2.17 & $\cdots$ & ISP/HSP \\
\hline 1FGL J0953.0-0838 & $2.22 \pm 0.40$ & $1.93 \pm 0.08$ & $\cdots$ & $\ldots$ & $\cdots$ & 1.64 & 1.28 & HSP \\
\hline 1FGL J1000.9+2915 & $1.95 \pm 0.43$ & $2.14 \pm 0.11$ & 0.558 & $\cdots$ & $\cdots$ & $\cdots$ & $\cdots$ & ISP \\
\hline 1FGL J1007.9+0619 & $3.02 \pm 0.70$ & $2.38 \pm 0.12$ & $\cdots$ & $\cdots$ & $\cdots$ & 2.17 & 1.44 & ISP \\
\hline 1FGL J1012.2+0634 & $1.61 \pm 0.76$ & $2.31 \pm 0.21$ & 0.727 & $\cdots$ & 0.52 & 2.16 & $\ldots$ & ISP \\
\hline 1FGL J1015.1+4927 & $6.44 \pm 0.48$ & $1.92 \pm 0.04$ & 0.212 & $\cdots$ & $\cdots$ & $\cdots$ & $\cdots$ & HSP \\
\hline 1FGL J1031.0+5051 & $0.57 \pm 0.23$ & $1.78 \pm 0.16$ & $\cdots$ & $\cdots$ & $\cdots$ & 2.17 & $\cdots$ & HSP \\
\hline 1FGL J1032.7+3737 & $1.38 \pm 0.42$ & $2.27 \pm 0.16$ & $\cdots$ & $\cdots$ & 0.53 & 2.17 & $\cdots$ & ISP \\
\hline 1FGL J1037.7+5711 & $3.22 \pm 0.47$ & $2.03 \pm 0.07$ & $\cdots$ & $\cdots$ & $\cdots$ & 1.64 & $\cdots$ & ISP \\
\hline 1FGL J1053.6+4927 & $0.41 \pm 0.14$ & $1.56 \pm 0.13$ & 0.140 & $\cdots$ & $\cdots$ & 2.17 & $\cdots$ & HSP \\
\hline 1FGL J1054.5+2212 & $3.67 \pm 0.13$ & $2.32 \pm 0.02$ & $\cdots$ & $\cdots$ & $\cdots$ & 1.64 & 1.36 & ISP \\
\hline 1FGL J1058.1-8006 & $7.50 \pm 0.43$ & $2.56 \pm 0.02$ & 0.581 & $\cdots$ & $\cdots$ & $\cdots$ & $\cdots$ & ISP \\
\hline 1FGL J1058.4+0134 & $13.88 \pm 0.07$ & $2.32 \pm 0.00$ & 0.888 & $\cdots$ & $\cdots$ & $\cdots$ & $\cdots$ & ISP \\
\hline 1FGL J1058.6+5628 & $5.62 \pm 0.53$ & $2.01 \pm 0.05$ & 0.143 & $\cdots$ & $\cdots$ & 2.18 & $\cdots$ & HSP \\
\hline 1FGL J1059.3-1132 & $4.37 \pm 0.02$ & $2.23 \pm 0.00$ & $\cdots$ & $\cdots$ & $\cdots$ & 1.65 & $\cdots$ & ISP \\
\hline 1FGL J1104.4+0734 & $2.17 \pm 0.56$ & $2.30 \pm 0.13$ & $\cdots$ & $\cdots$ & $\cdots$ & 1.65 & $\cdots$ & ISP/HSP \\
\hline 1FGL J1104.4+3812 & $17.09 \pm 0.57$ & $1.81 \pm 0.02$ & 0.031 & $\cdots$ & $\cdots$ & $\cdots$ & $\cdots$ & HSP \\
\hline 1FGL J1107.8+1502 & $0.86 \pm 0.04$ & $2.01 \pm 0.02$ & $\cdots$ & $\cdots$ & 0.60 & 2.16 & $\cdots$ & HSP \\
\hline 1FGL J1117.1+2013 & $1.36 \pm 0.27$ & $1.77 \pm 0.08$ & 0.138 & $\cdots$ & $\cdots$ & 2.17 & $\cdots$ & HSP \\
\hline 1FGL J1121.0+4209 & $0.39 \pm 0.16$ & $1.64 \pm 0.15$ & 0.124 & $\ldots$ & $\cdots$ & 2.17 & $\cdots$ & HSP \\
\hline 1FGL J1133.1+0033 & $2.66 \pm 0.52$ & $2.15 \pm 0.10$ & 0.678 & $\cdots$ & $\cdots$ & 1.86 & $\cdots$ & ISP \\
\hline 1FGL J1136.6+7009 & $1.14 \pm 0.27$ & $1.87 \pm 0.10$ & 0.046 & $\cdots$ & $\cdots$ & $\cdots$ & $\cdots$ & HSP \\
\hline 1FGL J1150.2+2419 & $2.10 \pm 0.50$ & $2.28 \pm 0.13$ & $\ldots$ & $\cdots$ & $\cdots$ & 2.21 & $\cdots$ & ISP \\
\hline 1FGL J1150.5+4152 & $1.76 \pm 0.37$ & $1.93 \pm 0.10$ & $\cdots$ & $\cdots$ & 0.85 & 1.66 & $\cdots$ & HSP \\
\hline 1FGL J1151.6+5857 & $1.31 \pm 0.56$ & $2.23 \pm 0.19$ & $\cdots$ & $\cdots$ & $\cdots$ & 1.76 & $\cdots$ & ISP \\
\hline 1FGL J1154.0-0008 & $0.38 \pm 0.34$ & $1.72 \pm 0.33$ & 0.254 & $\cdots$ & $\cdots$ & 2.22 & $\cdots$ & HSP \\
\hline 1FGL J1202.9+6032 & $2.71 \pm 0.75$ & $2.44 \pm 0.16$ & 0.065 & $\cdots$ & $\cdots$ & 2.18 & $\cdots$ & ISP \\
\hline 1FGL J1204.4+1139 & $1.33 \pm 0.46$ & $2.23 \pm 0.17$ & 0.296 & $\cdots$ & $\cdots$ & 2.17 & $\cdots$ & HSP \\
\hline 1FGL J1217.7+3007 & $5.86 \pm 0.58$ & $1.98 \pm 0.05$ & 0.130 & $\cdots$ & $\cdots$ & $\cdots$ & $\cdots$ & HSP \\
\hline 1FGL J1218.4-0128 & $1.11 \pm 0.31$ & $1.96 \pm 0.12$ & $\cdots$ & $\cdots$ & 0.64 & 1.64 & 1.23 & ISP \\
\hline 1FGL J1221.3+3008 & $2.02 \pm 0.36$ & $1.76 \pm 0.07$ & 0.184 & $\cdots$ & $\cdots$ & 2.18 & $\cdots$ & HSP \\
\hline 1FGL J1221.5+2814 & $8.12 \pm 0.64$ & $2.09 \pm 0.04$ & 0.103 & $\ldots$ & $\cdots$ & 2.22 & $\cdots$ & ISP \\
\hline 1FGL J1226.7-1332 & $0.60 \pm 0.21$ & $1.74 \pm 0.13$ & $\cdots$ & $\cdots$ & $\cdots$ & 1.76 & $1.30^{\mathrm{i}}$ & ISP \\
\hline 1FGL J1230.4+2520 & $1.21 \pm 0.35$ & $2.07 \pm 0.13$ & 0.135 & $\cdots$ & $\cdots$ & 1.78 & $\cdots$ & ISP \\
\hline 1FGL J1231.6+2850 & $2.46 \pm 0.36$ & $1.94 \pm 0.07$ & 0.236 & $\cdots$ & $\cdots$ & 2.18 & $\cdots$ & HSP \\
\hline
\end{tabular}


Table 5

(Continued)

\begin{tabular}{|c|c|c|c|c|c|c|c|c|}
\hline NAME & $\operatorname{Flux}_{100}{ }^{\mathrm{a}}$ & Photon Index & $z^{\mathrm{b}}$ & Photo- $z^{\mathrm{c}}$ & $z_{L} L^{\mathrm{d}}$ & $z_{\mathrm{MAX}}{ }^{\mathrm{e}}$ & Photo- $z_{U} L^{\mathrm{f}}$ & SED CLASS \\
\hline 1FGL J1243.1+3627 & $1.25 \pm 0.28$ & $1.79 \pm 0.09$ & $\cdots$ & $\cdots$ & 0.48 & 1.77 & $\cdots$ & HSP \\
\hline 1FGL J1248.2+5820 & $6.35 \pm 0.61$ & $2.17 \pm 0.06$ & $\cdots$ & $\ldots$ & $\ldots$ & 1.64 & $\cdots$ & ISP \\
\hline 1FGL J1249.8+3706 & $0.54 \pm 0.21$ & $1.80 \pm 0.15$ & $\ldots$ & $\ldots$ & $\ldots$ & 2.19 & $\cdots$ & HSP \\
\hline 1FGL J1253.0+5301 & $3.74 \pm 0.51$ & $2.13 \pm 0.08$ & $\cdots$ & $\cdots$ & 0.66 & 1.64 & $\cdots$ & ISP \\
\hline 1FGL J1303.0+2433 & $4.88 \pm 0.52$ & $2.17 \pm 0.06$ & $\cdots$ & $\cdots$ & 0.77 & 1.69 & $\cdots$ & ISP \\
\hline 1FGL J1304.3-4352 & $3.85 \pm 0.64$ & $2.06 \pm 0.07$ & $\cdots$ & $\cdots$ & $\cdots$ & 2.12 & $1.30^{\mathrm{i}}$ & HSP \\
\hline 1FGL J1309.5+4304 & $1.45 \pm 0.32$ & $1.94 \pm 0.10$ & 0.691 & $\cdots$ & 0.69 & 1.80 & $\cdots$ & HSP \\
\hline 1FGL J1314.7+2346 & $1.76 \pm 0.40$ & $2.10 \pm 0.11$ & $\cdots$ & $\cdots$ & $\cdots$ & 4.68 & 1.30 & ISP \\
\hline 1FGL J1338.9+1153 & $1.17 \pm 0.05$ & $2.08 \pm 0.02$ & $\ldots$ & $1.61_{-0.10}^{+0.04 i}$ & 1.59 & 1.94 & $\ldots$ & ISP \\
\hline 1FGL J1351.5+1115 & $0.17 \pm 0.02$ & $1.49 \pm 0.04$ & $\ldots$ & $\ldots$ & 0.62 & 1.64 & 1.12 & HSP \\
\hline 1FGL J1418.3-0235 & $1.31 \pm 0.33$ & $1.88 \pm 0.10$ & $\cdots$ & $\cdots$ & $\cdots$ & 1.64 & 1.37 & HSP \\
\hline 1FGL J1421.0+5421 & $3.69 \pm 0.88$ & $2.76 \pm 0.17$ & 0.153 & $\cdots$ & $\cdots$ & $\cdots$ & $\cdots$ & ISP \\
\hline 1FGL J1425.0+3614 & $0.78 \pm 0.39$ & $2.05 \pm 0.20$ & $\cdots$ & $\ldots$ & $\ldots$ & 2.17 & $\cdots$ & ISP \\
\hline 1FGL J1426.9+2347 & $7.47 \pm 0.49$ & $1.85 \pm 0.03$ & $\ldots$ & $\ldots$ & $\ldots$ & 1.66 & 1.11 & HSP \\
\hline 1FGL J1428.7+4239 & $0.38 \pm 0.17$ & $1.60 \pm 0.16$ & 0.129 & $\cdots$ & $\cdots$ & 2.18 & $\cdots$ & HSP \\
\hline 1FGL J1437.0+5640 & $0.20 \pm 0.12$ & $1.46 \pm 0.21$ & $\cdots$ & $\cdots$ & $\cdots$ & 2.08 & $\cdots$ & HSP \\
\hline 1FGL J1440.9+0613 & $5.66 \pm 0.85$ & $2.63 \pm 0.11$ & $\cdots$ & $\cdots$ & 0.32 & 1.63 & 1.31 & ISP \\
\hline 1FGL J1442.8+1158 & $0.44 \pm 0.26$ & $1.73 \pm 0.23$ & 0.163 & $\ldots$ & $\cdots$ & 2.17 & $\cdots$ & HSP \\
\hline 1FGL J1444.0-3906 & $2.72 \pm 0.45$ & $1.90 \pm 0.07$ & $\ldots$ & $\ldots$ & $\ldots$ & 2.20 & $\cdots$ & HSP \\
\hline 1FGL J1447.9+3608 & $1.60 \pm 0.39$ & $1.99 \pm 0.11$ & $\ldots$ & $\ldots$ & 0.74 & 1.76 & $\ldots$ & HSP \\
\hline 1FGL J1454.6+5125 & $2.58 \pm 0.53$ & $2.30 \pm 0.10$ & $\ldots$ & $\ldots$ & $\ldots$ & 1.63 & $\ldots$ & ISP \\
\hline 1FGL J1501.1+2237 & $1.16 \pm 0.26$ & $1.77 \pm 0.09$ & 0.235 & $\ldots$ & $\ldots$ & 2.18 & $\cdots$ & HSP \\
\hline 1FGL J1503.5-1544 & $0.89 \pm 0.45$ & $1.79 \pm 0.19$ & $\cdots$ & $\cdots$ & 0.21 & 1.76 & $\cdots$ & HSP \\
\hline 1FGL J1505.1-3435 & $1.85 \pm 0.73$ & $2.19 \pm 0.17$ & $\cdots$ & $\cdots$ & 1.55 & 3.13 & $\cdots$ & ISP \\
\hline 1FGL J1517.8-2423 & $7.47 \pm 0.83$ & $2.13 \pm 0.06$ & 0.048 & $\cdots$ & $\cdots$ & $\cdots$ & $\cdots$ & ISP \\
\hline 1FGL J1521.0-0350 & $1.67 \pm 0.50$ & $2.04 \pm 0.13$ & $\ldots$ & $\ldots$ & 0.87 & 1.80 & $\cdots$ & HSP \\
\hline 1FGL J1522.6-2732 & $5.94 \pm 0.84$ & $2.30 \pm 0.08$ & 1.294 & $\cdots$ & $\cdots$ & $\cdots$ & $\cdots$ & ISP \\
\hline 1FGL J1542.9+6129 & $7.08 \pm 0.62$ & $2.16 \pm 0.05$ & $\cdots$ & $\cdots$ & $\cdots$ & 1.76 & $\cdots$ & ISP \\
\hline 1FGL J1548.7-2250 & $2.36 \pm 0.85$ & $2.19 \pm 0.16$ & 0.192 & $\cdots$ & $\cdots$ & 1.65 & $\cdots$ & HSP \\
\hline 1FGL J1553.5-3116 & $0.50 \pm 0.21$ & $1.71 \pm 0.14$ & $\cdots$ & $\ldots$ & $\ldots$ & 1.97 & $\ldots$ & HSP \\
\hline 1FGL J1555.7+1111 & $6.77 \pm 0.45$ & $1.68 \pm 0.03$ & $\cdots$ & $\cdots$ & $\ldots$ & 1.77 & 1.35 & HSP \\
\hline 1FGL J1558.9+5627 & $2.60 \pm 0.75$ & $2.19 \pm 0.14$ & 0.300 & $\ldots$ & 1.05 & 2.47 & $\cdots$ & ISP \\
\hline 1FGL J1607.1+1552 & $4.62 \pm 0.66$ & $2.32 \pm 0.08$ & 0.496 & $\ldots$ & $\cdots$ & $\cdots$ & $\cdots$ & ISP \\
\hline 1FGL J1643.5-0646 & $4.11 \pm 0.86$ & $2.27 \pm 0.10$ & 0.082 & $\cdots$ & $\cdots$ & 2.07 & $\cdots$ & HSP \\
\hline 1FGL J1649.6+5241 & $1.61 \pm 0.48$ & $2.16 \pm 0.14$ & $\cdots$ & $\cdots$ & $\cdots$ & 2.47 & $\cdots$ & $\cdots$ \\
\hline 1FGL J1653.9+3945 & $5.67 \pm 0.45$ & $1.81 \pm 0.04$ & 0.034 & $\cdots$ & $\cdots$ & $\cdots$ & $\cdots$ & HSP \\
\hline 1FGL J1719.2+1745 & $4.33 \pm 0.52$ & $2.02 \pm 0.06$ & $\ldots$ & $\cdots$ & $\ldots$ & 1.64 & $\cdots$ & ISP \\
\hline 1FGL J1725.0+1151 & $2.48 \pm 0.50$ & $1.89 \pm 0.09$ & $\cdots$ & $\cdots$ & $\cdots$ & 1.65 & $\cdots$ & HSP \\
\hline 1FGL J1725.5+5854 & $1.31 \pm 0.35$ & $2.03 \pm 0.12$ & $\cdots$ & $\cdots$ & $\cdots$ & 1.66 & $\cdots$ & ISP \\
\hline 1FGL J1727.9+5010 & $0.79 \pm 0.33$ & $1.94 \pm 0.17$ & 0.055 & $\cdots$ & $\cdots$ & $\cdots$ & $\cdots$ & HSP \\
\hline 1FGL J1744.2+1934 & $0.74 \pm 0.32$ & $1.83 \pm 0.16$ & 0.083 & $\cdots$ & $\cdots$ & $\cdots$ & $\cdots$ & HSP \\
\hline 1FGL J1748.5+7004 & $2.29 \pm 0.20$ & $2.05 \pm 0.04$ & 0.770 & $\cdots$ & $\ldots$ & $\cdots$ & $\cdots$ & ISP \\
\hline 1FGL J1749.0+4323 & $2.39 \pm 0.10$ & $2.09 \pm 0.02$ & $\cdots$ & $\ldots$ & 0.57 & 1.65 & $\cdots$ & ISP \\
\hline 1FGL J1751.5+0937 & $11.15 \pm 1.37$ & $2.32 \pm 0.06$ & 0.322 & $\cdots$ & $\cdots$ & $\cdots$ & $\cdots$ & ISP \\
\hline 1FGL J1754.3+3212 & $3.06 \pm 0.53$ & $2.10 \pm 0.09$ & $\cdots$ & $\cdots$ & $\ldots$ & 1.63 & $\cdots$ & HSP \\
\hline 1FGL J1800.4+7827 & $6.11 \pm 0.04$ & $2.35 \pm 0.00$ & 0.684 & $\ldots$ & $\ldots$ & $\cdots$ & $\cdots$ & ISP \\
\hline 1FGL J1807.0+6945 & $6.33 \pm 0.89$ & $2.53 \pm 0.09$ & 0.051 & $\cdots$ & $\cdots$ & $\cdots$ & $\cdots$ & ISP \\
\hline 1FGL J1809.6+2908 & $1.20 \pm 0.47$ & $2.07 \pm 0.16$ & $\cdots$ & $\cdots$ & $\cdots$ & 1.63 & $\cdots$ & ISP \\
\hline 1FGL J1811.0+1607 & $3.35 \pm 0.69$ & $2.22 \pm 0.10$ & $\cdots$ & $\cdots$ & $\ldots$ & 1.74 & $\cdots$ & ISP \\
\hline 1FGL J1813.4+3141 & $2.77 \pm 0.49$ & $2.11 \pm 0.09$ & 0.117 & $\cdots$ & $\cdots$ & $\cdots$ & $\cdots$ & ISP \\
\hline 1FGL J1824.0+5651 & $6.55 \pm 0.73$ & $2.36 \pm 0.07$ & 0.664 & $\cdots$ & $\cdots$ & 2.48 & $\cdots$ & ISP \\
\hline 1FGL J1829.8+5404 & $1.96 \pm 0.71$ & $2.39 \pm 0.19$ & $\cdots$ & $\cdots$ & $\cdots$ & 2.46 & $\cdots$ & HSP \\
\hline 1FGL J1832.6-5700 & $2.40 \pm 0.74$ & $2.22 \pm 0.15$ & $\cdots$ & $\cdots$ & 1.23 & 1.96 & $\cdots$ & HSP \\
\hline 1FGL J1838.6+4756 & $1.09 \pm 0.36$ & $1.92 \pm 0.13$ & $\cdots$ & $\cdots$ & $\cdots$ & $\cdots$ & $\cdots$ & HSP \\
\hline 1FGL J1849.6-4314 & $2.04 \pm 0.56$ & $2.17 \pm 0.13$ & $\ldots$ & $\ldots$ & $\ldots$ & 1.94 & $\ldots$ & ISP/HSP \\
\hline 1FGL J1903.0+5539 & $2.93 \pm 0.46$ & $1.97 \pm 0.07$ & $\ldots$ & $\ldots$ & 0.73 & 1.63 & $\ldots$ & ISP \\
\hline 1FGL J1918.4-4108 & $2.06 \pm 0.42$ & $1.91 \pm 0.09$ & $\cdots$ & $\cdots$ & 1.59 & 2.11 & $\cdots$ & ISP \\
\hline 1FGL J1926.8+6153 & $2.76 \pm 0.55$ & $2.13 \pm 0.10$ & $\cdots$ & $\ldots$ & $\ldots$ & 1.65 & $\cdots$ & HSP \\
\hline 1FGL J1936.9-4720 & $0.73 \pm 0.36$ & $1.82 \pm 0.18$ & 0.265 & $\cdots$ & $\cdots$ & $\cdots$ & $\cdots$ & HSP \\
\hline 1FGL J1958.4-3013 & $2.07 \pm 0.82$ & $2.23 \pm 0.17$ & 0.119 & $\cdots$ & $\cdots$ & $\cdots$ & $\cdots$ & HSP \\
\hline 1FGL J2000.0+6508 & $7.22 \pm 0.67$ & $2.05 \pm 0.05$ & 0.049 & $\cdots$ & $\ldots$ & $\cdots$ & $\ldots$ & HSP \\
\hline 1FGL J2006.0+7751 & $3.14 \pm 0.91$ & $2.44 \pm 0.16$ & 0.342 & $\cdots$ & $\cdots$ & $\cdots$ & $\cdots$ & ISP \\
\hline 1FGL J2009.1+7228 & $4.32 \pm 1.15$ & $2.58 \pm 0.15$ & $\cdots$ & $\cdots$ & 1.74 & 2.03 & $\cdots$ & ISP \\
\hline 1FGL J2009.5-4849 & $3.87 \pm 0.49$ & $1.88 \pm 0.06$ & 0.071 & $\cdots$ & $\cdots$ & $\cdots$ & $\cdots$ & HSP \\
\hline 1FGL J2015.3-0129 & $2.26 \pm 0.62$ & $2.19 \pm 0.13$ & $\cdots$ & $\cdots$ & $\cdots$ & 1.78 & 1.22 & ISP \\
\hline 1FGL J2016.2-0903 & $2.21 \pm 0.01$ & $2.18 \pm 0.00$ & $\ldots$ & $\ldots$ & 0.60 & 1.63 & $\cdots$ & ISP \\
\hline 1FGL J2031.5+1219 & $4.11 \pm 0.04$ & $2.42 \pm 0.01$ & 1.213 & $\cdots$ & 0.85 & $\ldots$ & $\cdots$ & ISP \\
\hline
\end{tabular}


Table 5

(Continued)

\begin{tabular}{|c|c|c|c|c|c|c|c|c|}
\hline NAME & $\operatorname{Flux}_{100^{a}}$ & Photon Index & $z^{\mathrm{b}}$ & Photo- $z^{\mathrm{c}}$ & $z_{L} L^{\mathrm{d}}$ & $z_{\mathrm{MAX}}{ }^{\mathrm{e}}$ & Photo- $z_{U} L^{\mathrm{f}}$ & SED CLASS ${ }^{\mathrm{g}}$ \\
\hline 1FGL J2039.0-1047 & $2.80 \pm 0.12$ & $2.18 \pm 0.02$ & $\cdots$ & $\cdots$ & $\ldots$ & 1.63 & $\cdots$ & ISP \\
\hline 1FGL J2131.7-0914 & $0.88 \pm 0.39$ & $1.97 \pm 0.18$ & 0.449 & $\cdots$ & $\ldots$ & $\cdots$ & $\cdots$ & HSP \\
\hline 1FGL J2139.3-4235 & $9.71 \pm 0.69$ & $2.12 \pm 0.04$ & $\cdots$ & $\cdots$ & $\cdots$ & 1.91 & $\cdots$ & ISP \\
\hline 1FGL J2143.1-3927 & $1.34 \pm 0.48$ & $2.07 \pm 0.16$ & 0.429 & $\cdots$ & $\cdots$ & 2.00 & $\cdots$ & ISP/HSP \\
\hline 1FGL J2146.6-1345 & $1.09 \pm 0.35$ & $1.85 \pm 0.13$ & $\ldots$ & $\ldots$ & $\ldots$ & 1.64 & $\ldots$ & HSP \\
\hline 1FGL J2149.7+0327 & $3.19 \pm 0.82$ & $2.60 \pm 0.16$ & $\cdots$ & $\cdots$ & 0.72 & 1.62 & 1.42 & ISP \\
\hline 1FGL J2158.8-3013 & $21.73 \pm 0.71$ & $1.91 \pm 0.02$ & 0.116 & $\cdots$ & $\ldots$ & .. & $\cdots$ & HSP \\
\hline 1FGL J2223.3+0103 & $0.46 \pm 0.26$ & $1.85 \pm 0.21$ & & $\cdots$ & $\cdots$ & 1.63 & $\cdots$ & \\
\hline 1FGL J2236.2+2828 & $10.57 \pm 0.79$ & $2.38 \pm 0.05$ & 0.790 & $\ldots$ & $\cdots$ & 1.64 & $\cdots$ & ISP \\
\hline 1FGL J2236.4-1432 & $6.93 \pm 0.71$ & $2.37 \pm 0.07$ & $\cdots$ & $\cdots$ & 0.61 & 2.53 & 1.55 & ISP \\
\hline 1FGL J2243.1-2541 & $2.90 \pm 0.52$ & $2.27 \pm 0.10$ & 0.774 & $\ldots$ & $\ldots$ & $\cdots$ & $\cdots$ & ISP \\
\hline 1FGL J2244.0+2021 & $3.06 \pm 0.43$ & $1.90 \pm 0.07$ & $\cdots$ & $\cdots$ & 0.40 & 1.64 & $\cdots$ & HSP \\
\hline 1FGL J2247.3+0000 & $1.19 \pm 0.37$ & $2.08 \pm 0.14$ & 0.949 & $\cdots$ & $\cdots$ & 1.85 & $\cdots$ & ISP \\
\hline 1FGL J2250.1+3825 & $0.98 \pm 0.27$ & $1.80 \pm 0.10$ & 0.119 & $\cdots$ & $\cdots$ & $\cdots$ & $\cdots$ & HSP \\
\hline 1FGL J2251.7+4030 & $3.98 \pm 0.81$ & $2.45 \pm 0.11$ & 0.229 & $\cdots$ & $\cdots$ & 1.67 & $\cdots$ & ISP \\
\hline 1FGL J2256.3-2009 & $0.73 \pm 0.28$ & $1.95 \pm 0.16$ & $\cdots$ & $\cdots$ & $\cdots$ & 1.93 & $\cdots$ & ISP \\
\hline 1FGL J2307.3+1452 & $2.00 \pm 0.55$ & $2.16 \pm 0.13$ & $\cdots$ & $\cdots$ & $\cdots$ & 1.66 & $\cdots$ & ISP \\
\hline 1FGL J2323.5+4211 & $2.00 \pm 0.50$ & $1.97 \pm 0.11$ & $\cdots$ & $\cdots$ & 0.27 & 1.70 & $\cdots$ & HSP \\
\hline 1FGL J2325.2+3957 & $3.32 \pm 0.49$ & $2.03 \pm 0.07$ & $\cdots$ & $\cdots$ & 1.05 & 1.85 & $\cdots$ & ISP \\
\hline 1FGL J2325.8-4043 & $2.44 \pm 0.87$ & $2.22 \pm 0.15$ & $\cdots$ & $\cdots$ & $\cdots$ & $\cdots$ & $\cdots$ & HSP \\
\hline 1FGL J2329.2+3755 & $0.50 \pm 0.22$ & $1.66 \pm 0.15$ & $\cdots$ & $\ldots$ & $\cdots$ & 1.76 & $\cdots$ & HSP \\
\hline 1FGL J2334.7+1429 & $0.80 \pm 0.05$ & $2.04 \pm 0.02$ & $\cdots$ & $\ldots$ & $\cdots$ & 2.66 & $1.30^{\mathrm{i}}$ & ISP \\
\hline 1FGL J2339.0+2123 & $0.23 \pm 0.15$ & $1.57 \pm 0.23$ & 0.291 & $\ldots$ & $\cdots$ & $\ldots$ & $\cdots$ & HSP \\
\hline 1FGL J2341.6+8015 & $4.51 \pm 0.72$ & $2.23 \pm 0.08$ & 0.274 & $\cdots$ & $\cdots$ & $\ldots$ & $\cdots$ & HSP \\
\hline 1FGL J2343.6+3437 & $0.31 \pm 0.19$ & $1.68 \pm 0.22$ & 0.366 & $\cdots$ & $\cdots$ & $\cdots$ & $\cdots$ & HSP \\
\hline 1FGL J2352.1+1752 & $0.74 \pm 0.29$ & $1.96 \pm 0.16$ & $\cdots$ & 1.45 & 0.65 & 1.63 & $\cdots$ & HSP \\
\hline 1FGL J2359.0-3035 & $0.70 \pm 0.27$ & $1.95 \pm 0.16$ & 0.165 & $\ldots$ & $\ldots$ & $\ldots$ & $\cdots$ & HSP \\
\hline
\end{tabular}

Notes. The nature of the redshift constraints is described in Section 3.3.

${ }^{a}$ Flux in the $0.1-100 \mathrm{GeV}$ band in units of $10^{-8}$ photons $\mathrm{cm}^{-2} \mathrm{~s}^{-1}$.

${ }^{\mathrm{b}}$ Spectroscopic redshift as reported in Abdo et al. (2010d), Ackermann et al. (2011), Shaw et al. (2012), and Shaw et al. (2013b).

${ }^{\mathrm{c}}$ Photometric redshift estimates from Rau et al. (2012).

${ }^{\mathrm{d}}$ Spectroscopic redshift lower limits from Shaw et al. (2013b) and Shaw et al. (2013a).

${ }^{\mathrm{e}}$ Spectroscopic redshift upper limits from Shaw et al. (2013b).

${ }^{\mathrm{f}}$ Photometric redshift upper limits from Rau et al. (2012).

g Blazar classification based on the frequency of the peak of the synchrotron component as reported in Ackermann et al. (2011) and Shaw et al. (2013b).

${ }^{\mathrm{h}}$ From Pita et al. (2012).

${ }^{i}$ Photometric redshift or upper limits from the work of J. Bolmer et al. (2013, in preparation).

\section{A.2. Best-Fit Parameters to Subclasses of BL Lac Objects}

Tables 6 and 7 report the best-fit parameters to the HSP, ISP, and LSP subclasses as described in Sections 5.1 and 5.2.

Table 6

Best-Fit Parameters of the Pure Luminosity and Pure Density Evolution LFs to Subclasses of BL Lac Objects

\begin{tabular}{|c|c|c|c|c|c|c|c|c|c|c|c|}
\hline Model & $A^{\mathrm{a}}$ & $\gamma_{1}$ & $L_{*}^{\mathrm{b}}$ & $\gamma_{2}$ & $k$ & $\tau$ & $\xi$ & $\mu^{*}$ & $\beta$ & $\sigma$ & $-2 \ln L^{\mathrm{c}}$ \\
\hline PLE $_{H S P}$ & $7.40_{-3.37}^{+9.46} \times 10^{2}$ & $1.47_{-0.19}^{+0.88}$ & $6.45_{-2.94}^{+5.39} \times 10^{-2}$ & $7.62_{-5.94}^{+2.38}$ & $3.82_{-1.17}^{+1.29}$ & $1.35_{-0.33}^{+0.17}$ & $-0.41_{-0.14}^{+0.08}$ & $1.97_{-0.04}^{+0.09}$ & $4.47_{-3.79}^{+5.25} \times 10^{-2}$ & $0.25_{-0.03}^{+0.08}$ & -607.3 \\
\hline PLE $_{I S P+L S P}$ & $2.72_{-2.34}^{+6.93} \times 10^{2}$ & $1.60_{-0.31}^{+1.40}$ & $4.24_{-2.10}^{+7.23} \times 10^{-2}$ & $.08_{-2.24}^{+5.92}$ & $7.86_{-1.86}^{+1.41}$ & $0.98_{-0.32}^{+0.29}$ & $-0.25_{-0.09}^{+0.05}$ & $2.27_{-0.03}^{+0.04}$ & $-3.32_{-3.02}^{+2.46} \times 10^{-2}$ & $0.20_{-0.02}^{+0.03}$ & -272.0 \\
\hline PLE $_{L S P}$ & $86.57_{-58.31}^{+232.56}$ & $1.51_{-0.36}^{+0.77}$ & $8.05_{-4.34}^{+9.41} \times 10^{-2}$ & $8.14_{-5.26}^{+1.86}$ & $\begin{array}{l}7.59_{-2.09}^{+1.78} \\
\end{array}$ & $1.30_{-0.39}^{+0.26}$ & $-0.23_{-0.08}^{+0.05}$ & $2.32_{-0.08}^{+0.28}$ & $-3.23_{-7.25}^{+6.71} \times 10^{-2}$ & $0.23_{-0.04}^{+0.21}$ & -81.3 \\
\hline $\mathrm{PLE}_{\mathrm{HSP}+\mathrm{ISP}}$ & $1.22_{-0.55}^{+0.75} \times 10^{3}$ & $1.48_{-0.13}^{+0.15}$ & $3.68_{-1.14}^{+2.37} \times 10^{-2}$ & $5.39_{-1.32}^{+1.44}$ & $5.11_{-1.08}^{+1.03}$ & $1.26_{-0.21}^{+0.18}$ & $-0.34_{-0.09}^{+0.05}$ & $2.06_{-0.02}^{+0.03}$ & $4.86_{-1.90}^{+2.50} \times 10^{-2}$ & $0.25_{-0.02}^{+0.02}$ & -715.8 \\
\hline
\end{tabular}

Notes. Parameters without an error estimate were kept fixed during the fit. Parameter values were computed as the median of all the best-fit parameters to the Monte Carlo sample, while the uncertainties represent the $68 \%$ containment regions around the median value.

${ }^{\text {a }}$ In units of $10^{-13} \mathrm{Mpc}^{-3} \mathrm{erg}^{-1} \mathrm{~s}$.

${ }^{\mathrm{b}}$ In units of $10^{48} \mathrm{erg} \mathrm{s}^{-1}$.

c Value of the $-2 \times \log$-likelihood when the function is minimized. 
Best-Fit Parameters of the LDDE LFs to Subclasses of BL Lac Objects

\begin{tabular}{|c|c|c|c|c|c|c|c|c|c|c|c|c|c|}
\hline Model & $A^{\mathrm{a}}$ & $\gamma_{1}$ & $L_{*}{ }^{\mathrm{b}}$ & $\gamma_{2}$ & $z_{c}^{*}$ & $p 1^{*}$ & $\tau$ & $p 2$ & $\alpha$ & $\mu^{*}$ & $\bar{\beta}$ & $\sigma \sigma$ & $-2 \ln L^{\mathrm{c}}$ \\
\hline$\overline{\text { LDDE }_{H S P}}$ & $9.59_{-5.36}^{+11.77}$ & $0.28_{-0.29}^{+0.25}$ & $0.42_{-0.20}^{+0.26}$ & $3.47_{-1.20}^{+16.5}$ & $1.60_{-0.40}^{+0.20}$ & $0.48_{-0.48}^{+1.63}$ & $6.76_{-1.82}^{+2.33}$ & $-11.12_{-3.88}^{+6.10}$ & $0.11_{-0.08}^{+0.05}$ & $1.97_{-0.04}^{+0.09}$ & $4.40_{-355}^{+4.18} \times 10^{-2}$ & $0.24_{-0.04}^{+0.08}$ & $\overline{-619.4}$ \\
\hline $\mathrm{LDDE}_{\mathrm{ISP}+\mathrm{LSP}}$ & $17.1_{-14.5}^{+212.3}$ & $0.48_{-1.26}^{+0.36}$ & $0.45_{-0.42}^{+1.65}$ & $1.98_{-0.71}^{+10.49}$ & $1.15_{-0.20}^{+0.22}$ & $4.54_{-2.58}^{+2.64}$ & $3.82_{-1.61}^{+1.66}$ & $-5.89_{-3.81}^{+2.59}$ & $4.69_{-106.12}^{+68.47} \times 10^{-3}$ & $2.26_{-0.03}^{+0.04}$ & $-2.81_{-2.58}^{+2.21} \times 10^{-2}$ & $0.20_{-0.02}^{+0.03}$ & -275.8 \\
\hline LDDE $_{L S P}$ & $3.34_{-2.05}^{+36.99}$ & $0.48_{-0.67}^{+0.31}$ & $1.48_{-1.11}^{+0.70}$ & $6.33_{-4.91}^{+13.67}$ & $0.96_{-0.12}^{+0.30}$ & $4.10_{-3.35}^{+5.90}$ & $5.34_{-2.70}^{+4.66}$ & $-5.53_{-4.97}^{+2.12}$ & $-1.73_{-206.12}^{+93.76} \times 10^{-3}$ & $2.32_{-0.09}^{+0.20}$ & $-3.24_{-9.38}^{+7.53} \times 10^{-2}$ & $0.23_{-0.04}^{+0.21}$ & -87.7 \\
\hline $\mathrm{LDDE}_{\mathrm{HSP}+\mathrm{ISP}}$ & $29.1_{-16.0}^{+28.6}$ & $0.22_{-0.29}^{+0.24}$ & $0.26_{-0.13}^{+0.25}$ & $2.10_{-0.49}^{+1.09}$ & $1.46_{-0.18}^{+0.11}$ & $1.98_{-1.20}^{+1.45}$ & $6.38_{-1.66}^{+1.58}$ & $-8.29_{-5.28}^{+3.05}$ & $9.41_{-4.09}^{+3.81} \times 10^{-2}$ & $2.05_{-0.02}^{+0.03}$ & $5.55_{-2.17}^{+2.34} \times 10^{-2}$ & $0.24_{-0.02}^{+0.03}$ & -733.9 \\
\hline
\end{tabular}

Notes. Parameters without an error estimate were kept fixed during the fit. Parameter values were computed as the median of all the best-fit parameters to the Monte Carlo sample, while the uncertainties represent the $68 \%$ containment region around the median value.

${ }^{\text {a }}$ In unit of $10^{-10} \mathrm{Mpc}^{-3} \mathrm{erg}^{-1} \mathrm{~s}$.

${ }^{\mathrm{b}}$ In unit of $10^{48} \mathrm{erg} \mathrm{s}^{-1}$.

${ }^{c}$ Value of the $-2 \times \log$-likelihood when the function is minimized. 


\section{REFERENCES}

Abdo, A. A., Ackermann, M., Ajello, M., et al. 2010a, ApJS, 188, 405 Abdo, A. A., Ackermann, M., Ajello, M., et al. 2010b, PhRvL, 104, 101101 Abdo, A. A., Ackermann, M., Ajello, M., et al. 2010c, ApJ, 720, 435 Abdo, A. A., Ackermann, M., Ajello, M., et al. 2010d, ApJ, 715, 429 Ackermann, M., Ajello, M., Allafort, A., et al. 2011, ApJ, 743, 171 Ackermann, M., Ajello, M., Allafort, A., et al. 2012a, ApJ, 753, 83 Ackermann, M., Ajello, M., Allafort, A., et al. 2012b, Sci, 338, 1190 Ackermann, M., Ajello, M., Allafort, A., et al. 2013, ApJS, 209, 34 Ajello, M., Costamante, L., Sambruna, R. M., et al. 2009, ApJ, 699, 603 Ajello, M., Shaw, M. S., Romani, R. W., et al. 2012, ApJ, 751, 108 Akaike, H. 1974, ITAC, 19, 716

Atwood, W. B., Abdo, A. A., Ackermann, M., et al. 2009, ApJ, 697, 1071 Beckmann, V., Engels, D., Bade, N., \& Wucknitz, O. 2003, A\&A, 401, 927 Blandford, R. D., \& Rees, M. J. 1978, PhyS, 17, 265

Böttcher, M., \& Dermer, C. D. 2002, ApJ, 564, 86

Caccianiga, A., Maccacaro, T., Wolter, A., Della Ceca, R., \& Gioia, I. M. 2002, ApJ, 566, 181

Cara, M., \& Lister, M. L. 2008, ApJ, 674, 111

Cavaliere, A., \& D’Elia, V. 2002, ApJ, 571, 226

Costamante, L., Ghisellini, G., Giommi, P., et al. 2001, A\&A, 371, 512

D’Abrusco, R., Massaro, F., Ajello, M., et al. 2012, ApJ, 748, 68

Della Ceca, R., Caccianiga, A., Severgnini, P., et al. 2008, A\&A, 487, 119

Dermer, C. D., \& Schlickeiser, R. 1993, ApJ, 416, 458

Domínguez, A., Primack, J. R., Rosario, D. J., et al. 2011, MNRAS, 410, 2556

Dunlop, J. S., \& Peacock, J. A. 1990, MNRAS, 247, 19

Fichtel, C. E., Hartman, R. C., Kniffen, D. A., et al. 1975, ApJ, 198, 163

Finke, J. D. 2013, ApJ, 763, 134

Finke, J. D., Razzaque, S., \& Dermer, C. D. 2010, ApJ, 712, 238

Fossati, G., Maraschi, L., Celotti, A., Comastri, A., \& Ghisellini, G. 1998, MNRAS, 299, 433

Franceschini, A., Rodighiero, G., \& Vaccari, M. 2008, A\&A, 487, 837

Furniss, A., Williams, D. A., Danforth, C., et al. 2013, ApJL, 768, L31

Gehrels, N. 1986, ApJ, 303, 336

Ghisellini, G., Celotti, A., Fossati, G., Maraschi, L., \& Comastri, A. 1998, MNRAS, 301, 451

Ghisellini, G., Maraschi, L., \& Tavecchio, F. 2009, MNRAS, 396, L105

Ghisellini, G., Tavecchio, F., Foschini, L., et al. 2012, MNRAS, 425, 1371

Giommi, P., Menna, M. T., \& Padovani, P. 1999, MNRAS, 310, 465

Giommi, P., Padovani, P., \& Polenta, G. 2013, MNRAS, in press (arXiv:1302.4331)

Giommi, P., Padovani, P., Polenta, G., et al. 2012, MNRAS, 420, 2899

Hasinger, G., Miyaji, T., \& Schmidt, M. 2005, A\&A, 441, 417

Hogg, D. W. 1999, arXiv:astro-ph/9905116

La Franca, F., \& Cristiani, S. 1997, AJ, 113, 1517

La Franca, F., Fiore, F., Comastri, A., et al. 2005, ApJ, 635, 864

Lähteenmäki, A., \& Valtaoja, E. 2003, ApJ, 590, 95

Lister, M. L. 2003, ApJ, 599, 105

Lister, M. L., Homan, D. C., Kadler, M., et al. 2009, ApJL, 696, L22
Lister, M. L., \& Marscher, A. P. 1997, ApJ, 476, 572

Longair, M. S. 1966, MNRAS, 133, 421

Marcha, M. J. M., Browne, I. W. A., Impey, C. D., \& Smith, P. S. 1996, MNRAS, 281,425

Marcha, M. J. M., \& Caccianiga, A. 2013, MNRAS, 430, 246

Marshall, H. L., Tananbaum, H., Avni, Y., \& Zamorani, G. 1983, ApJ, 269, 35

Massaro, E., Giommi, P., Leto, C., et al. 2009, A\&A, 495, 691

Massaro, F., D’Abrusco, R., Tosti, G., et al. 2012, ApJ, 750, 138

Meyer, E. T., Fossati, G., Georganopoulos, M., \& Lister, M. L. 2012, ApJ, 752, L4

Miyaji, T., Hasinger, G., \& Schmidt, M. 2001, A\&A, 369, 49

Morganti, R., Oosterloo, T. A., Fosbury, R. A. E., \& Tadhunter, C. N. 1995, MNRAS, 274, 393

Narayan, R., Garcia, M. R., \& McClintock, J. E. 1997, ApJL, 478, L79

Padovani, P., Giommi, P., Landt, H., \& Perlman, E. S. 2007, ApJ, 662, 182

Padovani, P., Giommi, P., \& Rau, A. 2012, MNRAS, 422, L48

Petrov, L., Mahony, E. K., Edwards, P. G., Sadler, E. M., \& Schinzel, F. K. 2013, MNRAS, 432, 1294

Pita, S., Goldoni, P., Boisson, C., et al. 2012, in AIP Conf. Ser. 1505, High Energy Gamma-ray Astronomy, ed. F. A. Aharonian, W. Hofmann, \& F. M. Rieger (Melville, NY: AIP), 566

Rau, A., Schady, P., Greiner, J., et al. 2012, A\&A, 538, A26

Rector, T. A., Stocke, J. T., Perlman, E. S., Morris, S. L., \& Gioia, I. M. 2000, AJ, 120,1626

Savolainen, T., Homan, D. C., Hovatta, T., et al. 2010, A\&A, 512, A24

Sbarufatti, B., Treves, A., \& Falomo, R. 2005, ApJ, 635, 173

Schmidt, M. 1968, ApJ, 151, 393

Schmidt, M. 1972, ApJ, 176, 303

Shakura, N. I., \& Sunyaev, R. A. 1973, A\&A, 24, 337

Shaw, M. S., Filippenko, A. V., Romani, R. W., Cenko, S. B., \& Li, W. 2013a, AJ, 146,127

Shaw, M. S., Romani, R. W., Cotter, G., et al. 2012, ApJ, 748, 49

Shaw, M. S., Romani, R. W., Cotter, G., et al. 2013b, ApJ, 764, 135

Singal, J., Petrosian, V., \& Ajello, M. 2012, ApJ, 753, 45

Sreekumar, P., Bertsch, D. L., Dingus, B. L., et al. 1998, ApJ, 494, 523

Stickel, M., Padovani, P., Urry, C. M., Fried, J. W., \& Kuehr, H. 1991, ApJ, 374,431

Tavecchio, F., Ghisellini, G., Bonnoli, G., \& Foschini, L. 2011, MNRAS, 414, 3566

Urry, C. M., \& Padovani, P. 1991, ApJ, 371, 60

Urry, C. M., \& Padovani, P. 1995, PASP, 107, 803

Urry, C. M., Scarpa, R., O’Dowd, M., et al. 2000, ApJ, 532, 816

Urry, C. M., \& Shafer, R. A. 1984, ApJ, 280, 569

Wall, J. 2008, arXiv:0807.3792

Wall, J. V., \& Jenkins, C. R. 2012, Practical Statistics for Astronomers (Cambridge: Cambridge Univ. Press),

Wilks, S. S. 1938, Ann. Math. Stat., 9, 60

Willott, C. J., Rawlings, S., Blundell, K. M., Lacy, M., \& Eales, S. A. 2001, MNRAS, 322, 536 